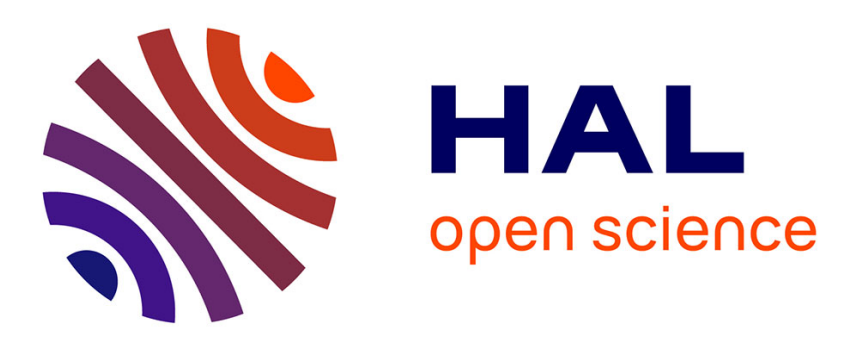

\title{
Inertial instability of intense stratified anticyclones. Part 1. Generalized stability criterion
}

\author{
Ayah Lazar, A. Stegner, E. Heifetz
}

\section{To cite this version:}

Ayah Lazar, A. Stegner, E. Heifetz. Inertial instability of intense stratified anticyclones. Part 1. Generalized stability criterion. Journal of Fluid Mechanics, 2013, 732 (october), pp.457-484. 10.1017/jfm.2013.412 . hal-01098651

\section{HAL Id: hal-01098651 https://hal.science/hal-01098651}

Submitted on 27 Dec 2014

HAL is a multi-disciplinary open access archive for the deposit and dissemination of scientific research documents, whether they are published or not. The documents may come from teaching and research institutions in France or abroad, or from public or private research centers.
L'archive ouverte pluridisciplinaire HAL, est destinée au dépôt et à la diffusion de documents scientifiques de niveau recherche, publiés ou non, émanant des établissements d'enseignement et de recherche français ou étrangers, des laboratoires publics ou privés. 


\title{
Inertial instability of intense stratified anticyclones. Part 1. Generalized stability criterion
}

\author{
Ayah Lazar ${ }^{1,2, \dagger}$, A. Stegner ${ }^{2}$ and E. Heifetz ${ }^{1,3}$ \\ ${ }^{1}$ Department of Geophysics and Planetary Science, \\ Tel Aviv University, Tel Aviv, Tel Aviv 69978, Israel \\ ${ }^{2}$ Laboratoire de Météorologie Dynamique, École Polytechnique, 91128 Palaiseau CEDEX, France \\ ${ }^{3}$ Department of Meteorology, Stockholm University, SE-106 91 Stockholm, Sweden
}

(Received 26 October 2012; revised 26 June 2013; accepted 7 August 2013)

The stability of axisymmetric vortices to inertial perturbations is investigated by means of linear stability analysis, taking into account stratification, vertical eddy viscosity, as well as finite depth of the flow. We consider different types of circular barotropic vortices in a linearly stratified shallow layer confined with rigid lids. For the simplest case of the Rankine vortex we develop an asymptotic analytic dispersion relation and a marginal stability criterion, which compares well with numerical results. This is a further generalization to the well-known generalized Rayleigh criterion, which is only valid for non-dissipative and non-stratified eddies. Unlike the Rayleigh criterion, it predicts that intense anticyclones may be stable even with a core region of negative absolute vorticity, and that the dissipation and stratification work together to stabilize the flow. Numerical analysis reveals that the stability diagrams for various types of vortices are almost identical in the Rossby, Burger and Ekman parameter space. This allows extension of our analytical solutions for the Rankine vortex to a wide variety of vortices. Furthermore, we show that a more suitable parameter for the intensity of the vortex is the vortex Rossby number, while for the inviscid case it is the local normalized vorticity. These predictions are in agreement with laboratory experiments presented in part 2 (J. Fluid Mech., vol. 732, 2013, pp. 485-509).

Key words: rotating flows, stratified flows, vortex instability

\section{Introduction}

Inertial instability of vortices is a centrifugal instability mechanism in the presence of the Coriolis force, when the equilibrium between the centrifugal, the radial pressure gradient and the Coriolis forces is unstable. It is a selective destructive mechanism that induces small three-dimensional perturbations on anticyclones with strong vorticity. For circular, barotropic and inviscid vortex columns a sufficient condition for instability to axisymmetric three-dimensional perturbations is that the square of the absolute angular momentum decreases with the radius, $r$, somewhere in the flow. This is equivalent to the generalized Rayleigh criterion (Kloosterziel \& van Heijst 
1991; Mutabazi, Normand \& Wesfreid 1992), which states that the Rayleigh discriminant should be negative somewhere in the flow $\chi(r) \equiv\left((1 / r) \partial_{r}(r V)+\right.$ $f)(2 V / r+f)<0$, where $V(r)$ is the azimuthal velocity and $f$ the Coriolis parameter. The unstable modes, similar to Taylor-Couette rolls, are located in the annular region surrounding the vortex core, where the Rayleigh discriminant is negative, as shown in laboratory experiments by Afanasyev \& Peltier (1998) and Afanasyev (2002) and numerical simulations by Orlandi \& Carnevale (1999) and Carnevale et al. (2011). In an unstratified fluid, small and intense anticyclones are strongly affected by such three-dimensional instabilities, as they break the cyclogeostrophic and the hydrostatic balance, triggering shear instability modes by enhancement of the vorticity gradients, and inducing a complete disruption of such vortices (Kloosterziel \& van Heijst 1991; Bartello, Métais \& Lesieur 1994; Orlandi \& Carnevale 1999; Carnevale et al. 2011). A criterion for general rotating and inviscid two-dimensional flows was derived by Leblanc \& Cambon (1997) and Sipp \& Jacquin (2000). In the framework of short wavelength perturbation this is a sufficient criterion for stability of circular vortices (identical to the Rayleigh criterion), but also for elliptical, hyperbolic and parallel flows.

This instability exhibits ultraviolet catastrophe (Smyth \& McWilliams 1998), i.e. the growth rate increases to an asymptotic value as vertical wavenumbers tend to infinity. In the absence of viscosity (which would ultimately limit the growth), this does not change as a result of stratification. On the contrary, stratification may even push the perturbations to smaller vertical wavelengths as a result of the potential energy barrier it creates. A short vertical wavelength allows us to apply the WKB method and extend the Rayleigh criterion for instability of axisymmetric vortices to non-axisymmetric perturbations. In this way, Billant \& Gallaire (2005) show for various vortices that the axisymmetric perturbations are generally more unstable than the non-axisymmetric vortices (this was also shown for a parallel shear by Griffiths 2008), indicating that the criterion is indeed a sufficient condition for inviscid three-dimensional instabilities. Furthermore, stratification does not change the criterion considerably.

However, as we shall show in this paper, when taking into account both stratification and dissipation, the generalized Rayleigh criterion greatly overestimates the unstable region in the parameter space. This is perfectly intuitive, as on the one hand, the stratification induces a low wavenumber cutoff because vertical velocities are inhibited, working against a stronger potential energy (confining the instability to smaller wavelengths), while on the other hand, viscosity eliminates the very short wavelengths. (This was shown in fully nonlinear simulations for barotropic vortices by Kloosterziel, Carnevale \& Orlandi 2007.) Thus, together these effects select the vertical wavenumber of the most unstable mode, and when the long- and short-wave cutoffs overlap, it may stabilize the flow altogether. In geophysical flows, where rotation, stratification and viscosity are all essential ingredients, these effects may explain the relative stability of intense anticyclones observed, for instance, in the lee of the Hawaii archipelago (Chavanne, Flament \& Gurgel 2010).

The above-mentioned effects of viscous dissipation and stratification have previously been studied thoroughly for the case of a parallel shear (Kloosterziel \& Carnevale 2008; Plougonven \& Zeitlin 2009). In this case, however, inertial instability is the unstable equilibrium between the Coriolis and the pressure gradient force alone, which might have a significant impact on the instability characteristics. Although the mechanisms are apparently similar in both configurations, there are significant qualitative differences between them, brought about by adding curvature to the problem. Even in the simple inviscid case, in a parallel shear, the instability occurs 
where $f Q<0$ ( $Q$ is the absolute vorticity), which means that it occurs where the absolute vorticity is the opposite sign of the Coriolis parameter (Johnson 1963; Stone 1966), whereas in the circular case, as mentioned above, it is where $\chi(r)<0$, which occurs immediately outside the negative absolute vorticity region. This means that in the linear growth stage, in the parallel shear case the instability acts to reduce $|Q|$, whereas it does not affect the core vorticity in the circular case. This is a major difference between the two cases, and there is no reason to believe that everything else is the same. For instance, according to numerical experiments conducted in Kloosterziel et al. (2007), the vertical wavenumber of the most unstable mode for a series of barotropic vortices scales with the stratification as $m \propto N^{1 / 5}$ (when $\operatorname{Re}=10^{4}$ ), whereas for a case of a barotropic linear shear it scales as $m \propto N^{1 / 2}$ for asymptotically large $\operatorname{Re}$ (Kloosterziel \& Carnevale 2008). Therefore, the findings for the parallel shear cannot be directly transposed to circular eddies.

The main goal of this paper is to quantify the parameter range for the instability of vortices to inertial perturbations, taking into account the stratification and eddy viscosity, and comparing it to the classical inviscid generalized Rayleigh criterion. First, we identify the relevant dynamical parameters which control the instability. We perform a linear stability analysis of barotropic circular vortices confined within a shallow layer. Starting with the stability analysis of the Rankine vortex ( $\$ 3)$, for which we provide a full analytical solution of the growth rate, we show that stabilization is significant only when stratification and dissipation work together, and a new stability criterion is derived (\$3.4). Then we show that this stability criterion is valid for various types of vortices $(\$ 4)$ when considering the stability in the parameter space of the Burger number, the vertical Ekman number and the vortex Rossby number (instead of the normalized core relative vorticity, which is often used for this purpose). Finally, we summarize our results and discuss the validity of this analysis for laboratory experiments and oceanic flows in $\S 5$.

\section{Circular vortices in a shallow layer: model and simplifying assumptions}

We assume a circular barotropic vortex confined in a linearly stratified surface layer with free-slip rigid lid at the top and bottom boundaries (see figure 1). Isopycnal tilting is neglected, and thus the baroclinic effects are ignored. We will refer to the limitations of this simplification to realistic GFD vortices later in the discussion (§5) and in appendix C. The general governing equations in cylindrical coordinates (on the $f$-plane, with the Boussinesq approximation) are given by

$$
\begin{gathered}
D_{t} u-\frac{v^{2}}{r}-f v=-\frac{1}{\rho_{0}} \partial_{r} p+v\left(\nabla^{2} u-\frac{u}{r^{2}}-\frac{2}{r^{2}} \partial_{\theta} v\right), \\
D_{t} v+\frac{u v}{r}+f u=-\frac{1}{\rho_{0} r} \partial_{\theta} p+v\left(\nabla^{2} v-\frac{v}{r^{2}}-\frac{2}{r^{2}} \partial_{\theta} u\right), \\
D_{t} w-b=-\frac{1}{\rho_{0}} \partial_{z} p+v \nabla^{2} w, \\
D_{t} b+N^{2} w=\frac{v}{P r} \nabla^{2} b,
\end{gathered}
$$

and

$$
\frac{1}{r} \partial_{r}(r u)+\frac{1}{r} \partial_{\theta} v+\partial_{z} w=0,
$$




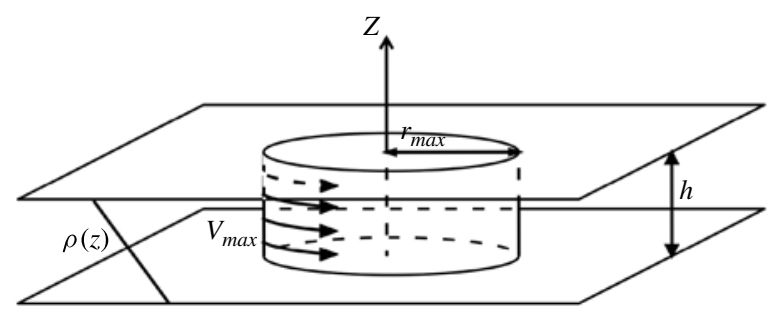

FIGURE 1. Model: a circular barotropic vortex in an idealized, linearly stratified surface layer, confined by free-slip rigid lids at the top and bottom. The isopycnal tilting is neglected.

where the material time derivative is $D_{t}=\partial_{t}+\boldsymbol{u} \cdot \nabla=\partial_{t}+u \partial_{r}+(v / r) \partial_{\theta}+w \partial_{z}$, $b=-g \rho^{\prime} / \rho_{0}$ is the buoyancy, $N^{2}=-g / \rho_{0} \partial_{z} \rho \overline{(z)}$ is the Brunt-Väisälä frequency, and $\operatorname{Pr}$ is the Prandtl number, which is the ratio between the kinematic viscosity $v$ and the diffusivity $\kappa$. In what follows, the Prandtl number is fixed to unity $\operatorname{Pr}=1(\kappa=v)$, which is a standard assertion for small-scale turbulent mixing and dissipation in oceanic and atmospheric flows. Equations (2.1)-(2.3) are the momentum equations for $(u, v, w)$, which are the velocities in the radial $(r)$, azimuthal $(\theta)$ and vertical $(z)$ directions, respectively. Equation (2.4) is the conservation of mass assuming an incompressible flow (equation (2.5)). The boundary conditions at the top and bottom are for vanishing normal velocity, $w(z=0)=w(z=h)=0$, and zero stress, $\partial_{z} u=\partial_{z} v=0$ (at $z=0, h$ as well). The radial boundary conditions are $u(r=0)=u(r \rightarrow+\infty) \rightarrow 0$.

In many geophysical flows such vortices are embedded within shallow layers, hence we assume a small vertical-to-horizontal aspect ratio. We therefore consider that the dissipation is mainly controlled by the vertical diffusivity, and thus the viscous term operator can be reduced to $\partial_{z}^{2}$. This assumption simplifies the system considerably, allowing for the linear stability analysis to be solved analytically for specific cases.

\section{1. linearized equations}

We consider barotropic mean flow, given by the velocity $\boldsymbol{U}=(0, V(r), 0)$ and the vertical vorticity, $\zeta(r)=1 / r \partial_{r}(r V)$. We introduce the perturbation field $\left(u^{\prime}, v^{\prime}, w^{\prime}, p^{\prime}, b^{\prime}\right)$ and linearize the Boussinesq equations (2.1)-(2.5) around the steady circular solution. Recent stability analysis (Gallaire \& Chomaz 2003; Billant \& Gallaire 2005) has shown that for centrifugally unstable vortices $(f=0)$, the symmetric $\left(\partial_{\theta}=0\right)$ modes are the most unstable ones. Numerical analysis of the effects of rotation and stratification on the stability of a barotropic axisymmetric vortex (Smyth \& McWilliams 1998) also showed that the axisymmetric mode is the most unstable for intense vortices. Given these results, we write down the linearized equations for purely axisymmetric mean flow and perturbations $\left(\partial_{\theta}=0\right)$ and get

$$
\begin{gathered}
\left(\partial_{t}-v \partial_{z}^{2}\right) u^{\prime}-\frac{2 v^{\prime} V(r)}{r}-f v^{\prime}=-\frac{1}{\rho_{0}} \partial_{r} p^{\prime}, \\
\left(\partial_{t}-v \partial_{z}^{2}\right) v^{\prime}+(\zeta(r)+f) u^{\prime}=0, \\
H y\left(\partial_{t}-v \partial_{z}^{2}\right) w^{\prime}-b^{\prime}=-\frac{1}{\rho_{0}} \partial_{z} p^{\prime}, \\
\left(\partial_{t}-v \partial_{z}^{2}\right) b^{\prime}+N^{2} w^{\prime}=0,
\end{gathered}
$$


and

$$
\frac{1}{r} \partial_{r}\left(r u^{\prime}\right)+\partial_{z} w^{\prime}=0 .
$$

Here we have introduced the binary hydrostatic parameter $H y$. It is zero in the case of hydrostatic balance (valid for the strong stratification regime), and equal to one otherwise. The primes are hereafter omitted for convenience.

We differentiate (2.6) by $z$ and subtract the derivative by $r$ of (2.8), to obtain the meridional component of the vorticity $\left(q=\partial_{z} u-H y \partial_{r} w\right)$ and thus get rid of the pressure term. By differentiating again for the time and plugging in the radial derivative of (2.9) and the vertical derivative of (2.7), we get a second-order partial differential equation for the vorticity,

$$
\tilde{\partial}_{t}^{2} q=\tilde{\partial}_{t}^{2}\left[\partial_{z} u-H y \partial_{r} w\right]=-\chi(r) \partial_{z} u+N^{2} \partial_{r} w
$$

where $\tilde{\partial}_{t}=\partial_{t}-v \partial_{z}^{2}$, and $\chi$ is what is usually referred to as the generalized Rayleigh discriminant,

$$
\chi(r) \equiv(\zeta+f)\left(\frac{2 V}{r}+f\right)
$$

We can understand the roles of $\chi$ and $N^{2}$ heuristically from (2.11) if we assume that the vorticity is generated by only one of its components at a time. If the vorticity is mainly driven by the radial velocities, $\partial_{z} u$, (2.11) becomes $\tilde{\partial}_{t}{ }^{2}\left(\partial_{z} u\right)=-\chi(r) \partial_{z} u$. This is a wave equation if $\chi(r)>0$ (i.e. neutral modes, stable solution) and an exponential growth (or decay) equation if $\chi(r)<0$ (i.e. growth and decay modes, unstable solution). On the other hand, if the vorticity is mainly driven by the vertical velocities, $\partial_{r} w$, the vorticity equation becomes $\tilde{\partial}_{t}^{2}\left(\partial_{r} w\right)=-N^{2} \partial_{r} w$, which is a wave equation associated with the strength of the stable stratification, $N$. Hence, the stratification has a stabilizing effect, and the necessary condition for instability,

$$
\chi(r)<0,
$$

is the generalized Rayleigh criterion. For an inviscid version of (2.6)-(2.10), Kloosterziel et al. (2007) reach a quadratic invariant of the flow (equation (2.8) in their paper). Then, considering a statically stable background $\left(N^{2}>0\right)$, they show that if $\chi>0$ everywhere, the flow must be stable. But if $\chi<0$ in some region of the flow, instability may develop, since $u, v, w$ and $b$ can grow without violating the conservation of the invariant.

For a more rigorous derivation, we define the meridional stream function $\psi$,

$$
\begin{gathered}
u=\partial_{z} \psi, \\
w=-\frac{1}{r} \partial_{r}(r \psi),
\end{gathered}
$$

and assume a discrete spectrum of normal-mode solutions $\psi_{m}(r, z)=$ $\phi_{m}(r) \sin (m \pi z / h) \mathrm{e}^{-\mathrm{i} \omega t}$, which satisfy the top and bottom boundary conditions, where $m=1,2,3, \ldots$ is a positive integer index that corresponds to the vertical wavenumber. The complex frequency is $\omega=\omega_{\text {real }}+\mathrm{i} \sigma$, and we introduce the generalized frequency $\tilde{\omega}=\omega+\mathrm{i} v(m \pi / h)^{2}=\omega_{\text {real }}+\mathrm{i}\left(\sigma+v(m \pi / h)^{2}\right)=\omega_{\text {real }}+\mathrm{i} \tilde{\sigma}$ to include the dissipation. The radial boundary conditions translate to $\phi_{m}(0)=0$ and $\phi_{m}(r \rightarrow+\infty) \rightarrow 0$. 
Substituting this stream function into (2.11) we get one second-order differential equation for $\phi_{m}(r)$, which resembles the Eliassen-Sawyer equation:

$$
\left(N^{2}-H y \tilde{\omega}^{2}\right)\left(\partial_{r}^{2} \phi_{m}+\frac{1}{r} \partial_{r} \phi_{m}-\frac{1}{r^{2}} \phi_{m}\right)-\left(\chi(r)-\tilde{\omega}^{2}\right)(m \pi / h)^{2} \phi_{m}=0 .
$$

Then, multiplying (2.16) by $\phi_{m}^{*}$ and integrating from zero to infinity, we obtain

$$
\int_{0}^{\infty}\left[\left(N^{2}-H y \tilde{\omega}^{2}\right)\left|\frac{1}{r} \partial_{r}\left(r \phi_{m}\right)\right|^{2}+\left(\chi(r)-\tilde{\omega}^{2}\right)(m \pi / h)^{2}\left|\phi_{m}\right|^{2}\right] r \mathrm{~d} r=0 .
$$

The imaginary part of this integral equation implies that $\tilde{\omega}$ is either real or imaginary, in other words that either $\omega_{\text {real }}=0$, or $\tilde{\sigma}=\sigma+v(m \pi / h)^{2}=0$. Therefore we have either a growing (or decaying) standing wave solution with $\tilde{\sigma} \neq 0$, or a neutral wave solution $\left(\omega_{\text {real }} \neq 0, \tilde{\sigma}=0\right.$, so that $\left.\sigma=-v(m \pi / h)^{2}\right)$. For the former solution (stable/unstable standing wave), we get

$$
\int_{0}^{\infty}\left[\left(N^{2}+H y \tilde{\sigma}^{2}\right)\left|\frac{1}{r} \partial_{r}\left(r \phi_{m}\right)\right|^{2}+\left(\chi(r)+\tilde{\sigma}^{2}\right)(m \pi / h)^{2}\left|\phi_{m}\right|^{2}\right] r \mathrm{~d} r=0 .
$$

This means that $\chi$ must be negative somewhere in order to satisfy the integral equation (2.18) with a localized normal mode $\phi_{m}$. Hence, again, we recover here the generalized Rayleigh criterion for instability, $\chi(r)<0$, found with similar reasoning in Kloosterziel \& van Heijst (1991) and Mutabazi et al. (1992). Furthermore, the upper limit for the growth rate is $\tilde{\sigma}<\sqrt{|\chi|_{\max }}$, which is the maximum growth rate for the inviscid limit, and for viscous cases it is $\sigma_{\max }=\sqrt{|\chi|_{\max }}-v(m \pi / h)^{2}$.

According to the first-order Taylor expansion of the velocity around the centre $V(r) \simeq \partial_{r} V(0) r$, the Rayleigh discriminant is always positive at the vortex core $\left(\chi(0) \simeq\left(f+2 \partial_{r} V(0) / f\right)^{2}=\left(f+\zeta_{0}\right)^{2}\right)$, even if the absolute vorticity is negative $Q(0)=f+\zeta_{0}<0$. Therefore, the unstable perturbations grow within an annular region located at the vortex edge corresponding to $\chi(r)<0$, where the absolute vorticity is $\sim f$ (Carnevale et al. 1997; Orlandi \& Carnevale 1999; Billant \& Gallaire 2005; Kloosterziel et al. 2007). For vorticity profiles with global minimum vorticity at the core this is the area of the maximum of the absolute value of velocity, $V_{\max }$. This is unlike the barotropic parallel shear configuration (Johnson 1963; Stevens \& Ciesielski 1986; Yanase et al. 1993; Plougonven \& Zeitlin 2009), where the unstable inertial perturbations are located in the region of negative absolute vorticity. For a parallel shear with a zonal mean flow, $\boldsymbol{U}=(U(y), 0,0)$ and zonally symmetric perturbations, one reaches an equation similar to (2.16), only with $f Q(y)=f\left(f-\partial_{y} U(y)\right)$ instead of $\chi$ (see the Appendix by S. D. Griffiths in Kloosterziel \& Carnevale 2008). The maximum growth rate is then given by the square-root of the absolute value of the global minimum of $f Q$. This is essentially the same as the circular case, but discarding the curvature term in $\chi$. However, this means that here the disturbances are localized where $Q$ is extremal and act to diminish it, while in the circular case the unstable perturbations act on the edge of the vortex, changing the velocity profile, and leaving the core vorticity untouched at the linear stage of the instability. 


\subsection{Dynamical parameters}

Assuming $N^{2}-H y \tilde{\omega}^{2} \neq 0$, which simply means that we cannot assume a hydrostatic balance for the case of a non-stratified flow, (2.16) can be written as

$$
\left(r^{2} \partial_{r}^{2} \phi_{m}+r \partial_{r} \phi_{m}-\phi_{m}\right)-\left(\frac{m \pi}{h}\right)^{2} \frac{\chi(r)-\tilde{\omega}^{2}}{N^{2}-H y \tilde{\omega}^{2}} \phi_{m}=0 .
$$

We then non-dimensionalize the velocities by $\left|V_{\max }\right|$, the length scales by the corresponding radius, $r_{\max }$ (i.e. $\left.\left|V\left(r_{\max }\right)\right|=\max |V(r)|\right)$, and the time scale with the inverse Coriolis parameter, $1 / f$, and rewrite the equation for the function $\phi_{m}(\eta)$, where $\eta=r / r_{\text {max }}$ :

$$
\eta^{2} \phi_{m}^{\prime \prime}+\eta \phi_{m}^{\prime}-\left(1+(m \pi)^{2} \frac{\hat{\chi}(\eta)-\hat{\omega}^{2}}{B u-H y \delta^{2} \hat{\omega}^{2}} \eta^{2}\right) \phi_{m}=0,
$$

with $\phi^{\prime}=\partial_{\eta} \phi$ and $\phi^{\prime \prime}=\partial_{\eta}^{2} \phi$.

In the equation above we introduced a few non-dimensional terms: the Burger number, which is $B u=\left(R d / r_{\max }\right)^{2}$, where $R d=N h / f$ is the deformation radius; the aspect ratio, $\delta=h / r_{\max }$; the non-dimensional frequency $\hat{\omega}=\tilde{\omega} / f=\omega_{\text {real }} / f+\mathrm{i} \hat{\sigma}$, where $\hat{\sigma}=\tilde{\sigma} / f=\sigma / f+(m \pi)^{2} E k$, and $E k=v / f h^{2}$ is the vertical Ekman number; and the nondimensional Rayleigh discriminant $\hat{\chi}(\eta)=\chi(\eta) / f^{2}$. Since $\hat{\chi}=(\zeta / f+1)(2 V / r f+1)$, it can be non-dimensionalized using the normalized core vorticity $\zeta(0) / f \equiv \zeta_{0} / f$, or by what we will call the 'vortex Rossby number', which we define as $R o=V_{\max } / r_{\max } f$ (see for example (3.2)). The relation between $\zeta_{0} / f$ and $R o$ depends on the specific velocity profile of an eddy. For all the vorticity profiles we study, the magnitude of the core vorticity is higher than the vortex Rossby number and satisfies the relation $\left|\zeta_{0} / f\right| \geqslant 2|R o|$ (where for a Rankine vortex it is an equality). In $\S 4$ we show that normalization by the vortex Rossby number provides a better mean of comparison between vortices with different profiles.

Hence, four independent parameters govern the stability of barotropic vortices in a rotating and stratified viscid shallow layer: the aspect ratio, the Burger number, the vortex Rossby number (or alternatively the normalized core vorticity), and the vertical Ekman number. When $\delta$ is small, as is generally the case for geophysical flow, a hydrostatic balance is maintained. Equation (2.20) then approaches the hydrostatic limit without having to set $H y=0$ explicitly.

An additional important parameter is the Froude number, defined as the ratio of the flow velocity over the maximum phase speed of internal gravity waves. Supercritical vortices are unlikely to occur in geophysical fluid dynamics and therefore we restrict our stability analysis to the subcritical regime:

$$
F r=\frac{|V|_{\max }}{N h}=\frac{|R o|}{\sqrt{B u}}<1 .
$$

\section{Rankine vortex}

We start by solving (2.20) for a Rankine vortex:

$$
\frac{\zeta(\eta)}{f}= \begin{cases}\frac{\zeta_{0}}{f} & \eta=\frac{r}{r_{\max }}<1, \\ 0 & \eta>1,\end{cases}
$$

which leads to direct analytical solutions. The relative core vorticity is equal to twice the vortex Rossby number $\zeta_{0} / f=2 R o$, and the non-dimensional Rayleigh discriminant 
(shown by the solid line in figure $2 a$ ) is

$$
\chi(\eta) / f^{2}= \begin{cases}\left(1+\frac{\zeta_{0}}{f}\right)^{2}=(1+2 R o)^{2} & \eta<1, \\ 1+\frac{\zeta_{0}}{f} \frac{1}{\eta^{2}}=1+2 R o \frac{1}{\eta^{2}} & \eta>1\end{cases}
$$

Plugging this into (2.20) leads to two modified Bessel equations of an imaginary and real order, for inside and outside the vortex, respectively.

$$
\begin{cases}\eta^{2} \phi_{m}^{\prime \prime}+\eta \phi_{m}^{\prime}-\left(1+\alpha^{2} \eta^{2}\right) \phi_{m}=0 & \eta<1 \\ \eta^{2} \phi_{m}^{\prime \prime}+\eta \phi_{m}^{\prime}-\left(\gamma^{2}+\beta^{2} \eta^{2}\right) \phi_{m}=0 & \eta>1\end{cases}
$$

The parameters $\alpha, \beta$ and $\gamma$ are

$$
\begin{gathered}
\alpha^{2}=(m \pi)^{2} \frac{(1+2 R o)^{2}-\hat{\omega}^{2}}{B u-H y \delta^{2} \hat{\omega}^{2}}, \\
\beta^{2}=(m \pi)^{2} \frac{1-\hat{\omega}^{2}}{B u-H y \delta^{2} \hat{\omega}^{2}}, \\
\gamma^{2}=1+(m \pi)^{2} \frac{2 R o}{B u-H y \delta^{2} \hat{\omega}^{2}} .
\end{gathered}
$$

A modified Bessel equation of the order $\mu \in \operatorname{Re}>0$ (not necessarily an integer) for a function with the rescaled variable $\rho r \in \operatorname{Re}>0, \phi(\rho r)$ is $r^{2} \partial_{r}^{2} \phi+r \partial_{r} \phi-\left(\mu^{2}+\rho^{2} r^{2}\right)$ $\phi=0$, the solution of which is the superposition of the modified function from the first and second kind of order $\mu, \phi(\rho r)=a \mathrm{I}_{\mu}(\rho r)+b \mathrm{~K}_{\mu}(\rho r)$. These functions are real and monotonic; $\mathrm{I}_{\mu}$ is increasing with $r$, and $\mathrm{K}_{\mu}$ decreases with $r$ and goes to infinity as $r=0$. However, when $\mu \in \operatorname{Im}$ the functions are not necessarily real or monotonic. The function $\mathrm{K}_{\mu}(r)$ is real for positive real $r$, oscillating with an infinite number of zeros between $0<r<-\mathrm{i} \mu$, and has no zeros in $-\mathrm{i} \mu \leqslant r<\infty$ (for further details see Dunster 1990).

Taking into account the radial boundary conditions, we obtain the piecewise eigenmodes

$$
\phi_{m}(\eta)= \begin{cases}a \mathrm{I}_{1}(\alpha \eta) & \eta \leqslant 1, \\ b \mathrm{~K}_{\gamma}(\beta \eta) & \eta \geqslant 1,\end{cases}
$$

where $a$ and $b$ are constants, the ratio of which is determined by the requirement of continuity of the radial velocity, $u$, and its derivative. This translates to the continuity of $\phi$ and $\phi^{\prime}$, which gives at $\eta=1$ :

$$
\alpha \frac{\mathrm{I}_{1}^{\prime}(\alpha)}{\mathrm{I}_{1}(\alpha)}=\beta \frac{\mathrm{K}_{\gamma}^{\prime}(\beta)}{\mathrm{K}_{\gamma}(\beta)} .
$$

This implicit equation is solved with the software Mathematica to obtain its multiple roots $\hat{\omega}^{2}=\left(\omega_{\text {real }} / f+\mathrm{i} \hat{\sigma}\right)^{2}$ for every set of $R o, B u, \delta$ and $m$ (the Ekman number only enters in the equation for $\hat{\omega}$ ). The positive roots correspond to a continuous spectrum of inertial modes, with $\hat{\omega}^{2}=\left(\omega_{\text {real }} / f\right)^{2}$, and no growth. Here we focus on the discrete spectrum of negative roots $\hat{\omega}_{n}^{2}=-\hat{\sigma}_{n}^{2}$ (where $n=0,1,2,3, \ldots, n_{\text {max }}$ ), which correspond to the $n$ growing/decaying trapped standing waves, the eigenmodes $\phi_{n, m}$. Mode $\phi_{n, m}$ has $n$ horizontal nodes, and a corresponding growth rate of 

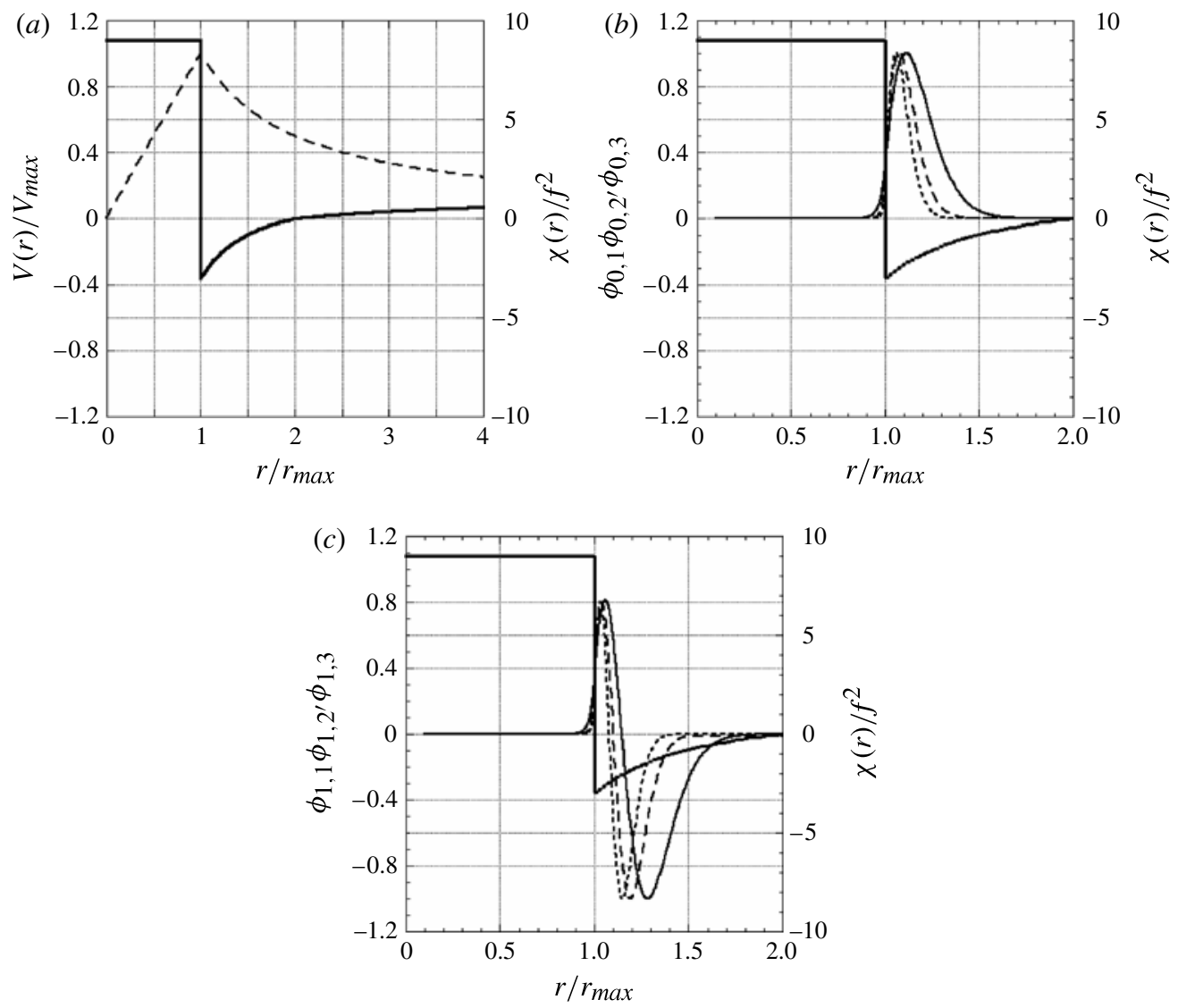

FIGURE 2. (a) The velocity profile (dashed line) and generalized Rayleigh discriminant (solid line) for a Rankine vortex with a vortex Rossby number $R o=-2$. ( $b$ ) The first radial eigenmodes $\phi_{0, m}\left(r / r_{\max }\right)$ corresponding to the parameter set $R o=-2, \delta=0.2$ and $B u=0$ $(S=N / f=0)$, for the first three vertical modes $m=1,2$ and 3 (solid, dashed and dotted lines, respectively), and the Rayleigh discriminant (heavy solid line). (c) The second horizontal mode, $\phi_{1, m}\left(r / r_{\max }\right)$, is delineated for the first three vertical modes $(m=1,2$ and 3 , solid, dashed and dotted lines, respectively), again along with the Rayleigh discriminant.

$\sigma_{n} / f= \pm \hat{\sigma}_{n}-(m \pi)^{2} E k$. The number $n$ is equivalent to a discrete radial wavenumber, and $n_{\max }$ is determined according to the number of positive solutions that exist for (3.8). The first radial mode, $\phi_{0, m}$, always has the largest growth, the second is the second largest and so on - i.e. $\hat{\sigma}_{0, m} \geqslant \hat{\sigma}_{1, m} \cdots \geqslant \hat{\sigma}_{n_{\max }, m} \geqslant 0$ (see $\S$ A.1 for more details).

We will explore the eigenmodes and growth rates in the parameter space defined by the vortex intensity (vortex Rossby number or the normalized core vorticity), the Burger number, the aspect ratio and the Ekman number. We begin by examining two extreme cases of zero and strong stratification.

\subsection{Unstratified case}

We consider the unstratified case $B u=0$, and plot in figure $2(b)$ the first radial eigenmodes $\phi_{0,1}, \phi_{0,2}, \phi_{0,3}$ and in figure $2(c)$ the second radial eigenmodes $\phi_{1,1}$, $\phi_{1,2}, \phi_{1,3}$ corresponding to the parameter set $R o=-2$ (intense anticyclone), $\delta=0.2$ (small but finite aspect ratio). As expected, these eigenmodes are localized at the 
vortex edge within the region $\chi(r)<0$. Also, with increasing vertical wavenumber the horizontal extent of the modes decreases. For the first vertical mode, $m=1$, and the parameters above, the first roots are $\hat{\sigma}_{0,1}^{2}=1.65$ and $\hat{\sigma}_{1,1}^{2}=1.05$ so that the first radial eigenmode $\phi_{0,1}$ will become unstable when $1 / E k>\pi^{2} / \hat{\sigma}_{0,1} \simeq 7.7$ while the other eigenmodes need larger values $1 / E k>\pi^{2} m^{2} / \hat{\sigma}_{n, m}>\pi^{2} / \hat{\sigma}_{0,1}$ to become unstable. Hence, for the unstratified case, the marginal stability limit will correspond to the curve $(1 / E k)_{c}=\pi^{2} / \sqrt{\hat{\sigma}_{0,1}}=F(R o, \delta)$. Such marginal stability curves are plotted in the $(R o, 1 / E k)$ parameter space for various values of the aspect ratio parameter $\delta$ in figure 3(a). In an inviscid flow a Rankine vortex is stable when the Rayleigh discriminant $\chi(r)$ is positive everywhere in the flow. In a Rankine vortex, this happens for a vortex Rossby number larger than $R o>R o_{c}=-0.5$ (which is equivalent to a core vorticity that is larger than the planetary vorticity, $\left.\zeta_{0} / f=2 R o>-1\right)$, marked by the shaded area in figure 3(a). The marginal stability curves for all aspect ratios are relatively flat for $R o<-0.5$, and are asymptotic to the inviscid limit for Rossby numbers close to $R o \approx R o_{c}$. They are very close to the inviscid limit for the whole range of $1 / E k>10$, even when the shallow water constraint is not satisfied $(\delta=1)$. Hence, for unstratified flow with weak dissipation $(1 / E k>10)$ the marginal stability limit is essentially the inviscid one, and the vortex stability depends primarily on its intensity $\left(R o>R o_{c}\right)$ and not on its size or even on its aspect ratio.

Note that the weak dependence of the marginal stability on the aspect ratio shown in figure 3(a) is slightly confusing, stemming from the parameter space that was chosen in order to compare with the strongly stratified case. As smaller vertical scales are expected to dissipate more strongly, it is expected that the smaller the aspect ratio the more stable the vortex, which seems to be the opposite of what is depicted. However, one must remember that the Ekman number has an implicit dependence on the aspect ratio. As $1 / E k=f h^{2} / \nu$ it should be expected that the larger $\delta=h / r_{\max }$ is, the higher it will be on this scale because it is squared while $v$ is only to the power of -1 .

\subsection{Strongly stratified regime}

In the strong stratification regime, $B u \gg \delta^{2} \hat{\omega}^{2}$, the stability diagrams are very different. This case corresponds to the hydrostatic approximation, in which case the hydrostatic parameter is set to zero, $H y=0$. The parameters relevant to the problem, along with the vortex Rossby number and the Ekman number, include the Burger number $B u=\left(R d / r_{\max }\right)^{2}$. The marginal stability curves for various Burger numbers, $B u=36$, $B u=4$ and $B u=1$, are plotted in figure 3(b), in comparison with the unstratified case $(B u=0)$. The line $B u=4$ is constructed by three different lines. The first two are in a non-hydrostatic approximation $(H y=1)$, one with $\delta=0.2$ and the other with $\delta=0.02$, and the third in the hydrostatic approximation $(H y=0)$. These fall directly one on top of the other, meaning that even for $B u=4$ the hydrostatic approximation is very accurate. Since stratification tends to favour small vertical scales, higher vertical modes ( $m=2$ or higher) may be more unstable in this case, especially when $v$ is small (the right upper part of the curves). This is the reason for the kinks in the curves with $B u \neq 0$. In $B u=1$, for instance, the vertical mode corresponding to maximal growth $\left(\sigma_{\max } / f=\sigma\left(m_{\max }\right) / f=0\right)$ changes from $m_{\max }=1$ to $m_{\max }=2$ around $R o=-1.5$. All the curves have kinks where the vertical modes 'jump', but when the modes are larger, as is the case for the larger Burger numbers, the difference is less pronounced. The non-stratified case $(B u=0)$ does not have any kinks because the vertical mode is always $m_{\max }=1$.

In contrast to figure $3(a)$, here (figure $3 b$ ) the change of the Burger number stabilizes the flow considerably (notice that the $1 / E k$ scale in this panel is much 

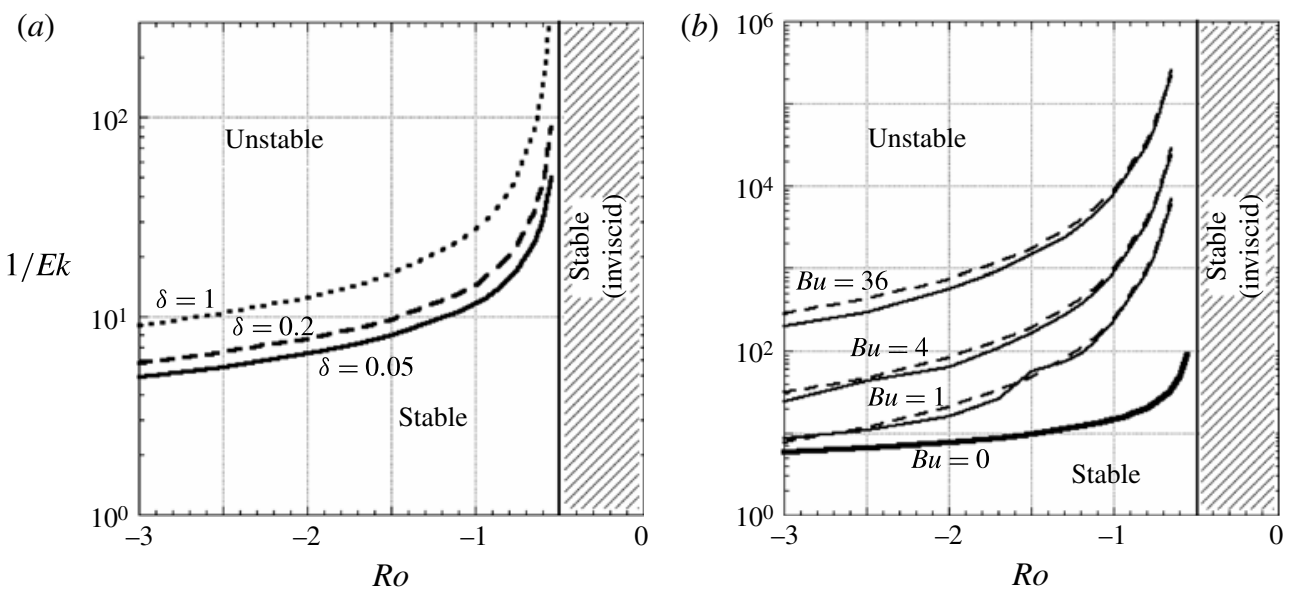

FIGURE 3. Marginal stability $(\sigma / f=0)$ diagrams for a Rankine vortex on the $(R o, 1 / E k)$ parameter space. These are compared to the inviscid Rayleigh criterion for stability for a Rankine vortex, $R o=-0.5$, shown in both panels as a shaded area, where $\chi(r)>0$ everywhere in the flow. (a) The effect of different aspect ratios, $\delta=h / r_{\max }$, on the stability of a Rankine vortex in a non-stratified fluid. The values of $\delta$ are indicated. $(b)$ The effect of the Burger number $\left(B u=\left(R d / r_{\max }\right)^{2}\right.$, indicated in the figure). The solid lines are the exact solutions calculated from (3.8) with Mathematica software and they are compared with the analytic approximation (dashed lines) given in (3.12).

larger than in figure $3 a$ ). The curves are not flat in the $R o<R o_{c}=-0.5$ range, as the stratification amplifies the stabilization effect of the dissipation, and the larger the Burger number, the larger the stable region in the $(R o, 1 / E k)$ parameter space. For submesoscale vortices $(B u>1)$, the marginal stability curves differ strongly from the inviscid limit even for weak vertical dissipation (i.e. $1 / E k \geqslant 1000$ ). In the inviscid limit $(1 / E k \rightarrow \infty)$ the marginal stability curves for all the stratified and the unstratified Rankine vortices tend to the asymptotic inviscid limit $R o_{c}=-0.5$, which means that it is not the stratification alone that stabilizes the inertial instability, but that it amplifies the stabilization induced by the vertical dissipation.

As one would expect, these findings are qualitatively the same for the case of a linear shear. This is reasonable, as the stratification and dissipation work in a similar way on the roll-up vortices created in the linear shear instability. Stratification stabilizes the flow because the perturbations need to work against a stronger potential energy, and dissipation diffuses them. For the linear shear, Kloosterziel \& Carnevale (2008) showed too that stronger shear requires a lower critical Reynolds number $(\propto 1 / E k)$, and that larger stratification $(\propto B u)$ requires a larger critical Reynolds number. We shall see to what extent the stability of the linear shear and the Rankine vortex differ quantitatively when we compute the new stability criterion for a Rankine vortex analytically in $\S \S 3.3$ and 3.4 .

\subsection{Growth rate behaviour}

To understand the origin of this enhanced stabilization we look more carefully at the dispersion relation of the inertially unstable modes. We study the impact of increasing dissipation (figure $4 a$ ) and of increasing Burger number (figure $4 b$ ) on the growth rate $\sigma / f=F(m)$ of the first radial eigenmode $\phi_{0, m}$. In viscous flows, small vertical 

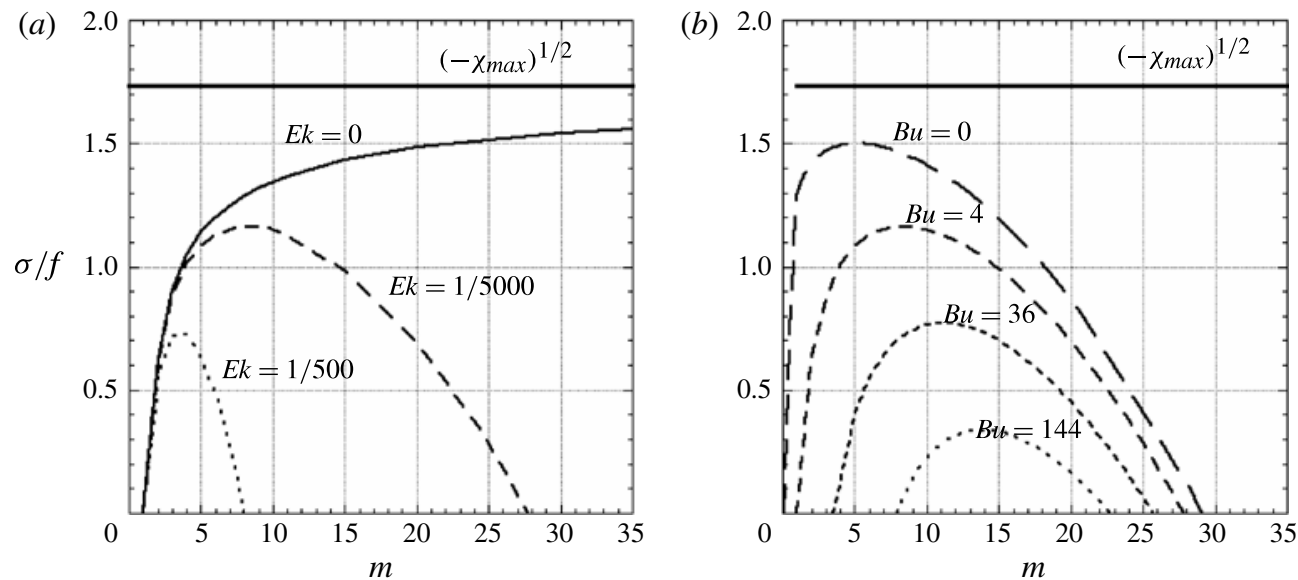

FIGURE 4. The growth rate $\sigma / f$ as a function of the vertical wavenumber, $m$ : $(a)$ the effect of the dissipation (the Ekman number, $E k) ;(b)$ the effect of the stratification (the Burger number, $B u$ ). The maximal asymptotic value for the growth rate, $\sqrt{|\chi|_{\max }}$, is plotted in both by a solid line. (a) $R o=-2, B u=4$; (b) $R o=-2, E k=1 / 5000$.

wavelength perturbations (large $m$ ) are stabilized by the vertical dissipation, leading to a cutoff $m<m_{E k}$, above which no growth occurs. This affects the vertical wavenumber $m_{\max }$ selected by the maximum growth rate $\left(\sigma\left(m_{\max }\right)=\max (\sigma)\right)$, as can be seen in figure 4(a), where the effect of increasing dissipation (increasing Ekman number) for the case of a Rankine vortex with $R o=-2$ and $B u=4$ is shown. Increasing the dissipation (from $1 / E k=5000$ to 500 ) leads to a smaller cutoff, which also leads to a smaller most unstable wavenumber, $m_{\max }=8$ and $m_{\max }=4$, respectively. This in turn also decreases the maximal growth rate, until the marginal stability limit is reached (at $1 / E k=63$, according to figure $3 b$ ).

On the other hand, stratification bounds the unstable wavenumbers from below, $m_{B u} \leqslant m$. This is because the vertical motion of the meridional circulation of the instability requires work in a stratified fluid, taking some of the energy that would otherwise be available for growth. This means that higher growth rate is associated with large vertical wavenumbers (which is then decreased by the dissipation). Figure 4(b) shows the dispersion relation for $R o=-2$ and $1 / E k=5000$, and increasing Burger numbers, $B u=0,4,36$ and 144, leading to an increase of $m_{\max }$ and a decay of the maximum growth rate. Even for this relatively small dissipation $(1 / E k=5000)$, a complete stabilization will be reached when the Burger number exceeds $\sqrt{B u} \approx 16$ (as would be evident by continuing the $\sigma T=0$ line in figure $10 a$ ), or in other words, when the vortices are smaller than $\sim R d / 16$. Notice that increasing stratification also leads to an even lower upper bound for $m$. This is to be expected, as stratification stabilizes all wavenumbers, so that the upper bound created by the dissipation is strengthened by adding stratification, which is how stratification and dissipation work together to amplify the effect of one another. In the absence of dissipation there would be no upper cutoff at all, so that the stratification would have less of an effect. These effects $\left(m_{B u}<m<m_{E k}\right)$ can be understood from analytical formulae (see appendix B).

Hence, in the strong stratification regime, where the unstable modes are constrained to large vertical wavenumbers, we may develop the roots of (3.8) for large $m$ 
and reach an approximate analytic term for the dispersion relation, of the form $\sigma / f \simeq F(R o)\left(1-\left(m_{c} / m\right)^{a}\right)$, for the instability of a Rankine vortex in the non-dissipative case (see appendix A). The small wavenumber cutoff is a function of the relative stratification, $B u$, and the relative rotation, $R o$, i.e. $m_{c}=G(R o, B u)$. Taking into account the vertical dissipation we get an asymptotic dispersion relation of the form $\sigma / f \simeq \sqrt{|\chi|_{\max }}\left(1-\left(m_{c} / m\right)^{a}\right)-(m \pi)^{2} E k$, which is

$$
\frac{\sigma}{f} \approx \sqrt{|2 R o+1|}\left(1+a_{s}\left(\frac{\sqrt{2}}{\pi}\right)^{2 / 3} \frac{|R o|^{2 / 3}}{|2 R o+1|} B u^{1 / 3} m^{-2 / 3}\right)-(m \pi)^{2} E k,
$$

where $a_{s}$ indicates the sth zero of $\mathrm{Ai}$, the Airy function, which is regular at infinity. We should take the first zero of the Airy function $a_{0}=-2.3381$ in order to get the first radial mode. A similar prediction for the dispersion relation is achieved by adapting Billant \& Gallaire's WKB approximation of the Taylor-Couette flow (Billant $\&$ Gallaire 2005) to a stratified flow (see again appendix A for more details).

The maximal growth rate is

$$
\left(\frac{\sigma}{f}\right)_{\max } \approx \sqrt{|2 R o+1|}\left(1-(B u E k)^{1 / 4} \frac{|R o|^{1 / 2}}{(\sqrt{|2 R o+1|})^{7 / 4}}\left[\frac{8\left|a_{0}\right|}{3}\right]^{3 / 4}\right),
$$

which shows that indeed the maximal growth rate is bounded by $\sqrt{|\chi|_{\max }}=$ $\sqrt{|2 R o+1|}$, as seen from (2.18), and increasing the stratification or the dissipation stabilizes the flow. This maximal growth is reached for the vertical wavenumber (the most unstable mode):

$$
m_{\max }=\frac{2^{1 / 8}\left|a_{0}\right|^{3 / 8}}{3^{3 / 8} \pi} B u^{\frac{1}{8}} E k^{-3 / 8} \frac{|R o|^{1 / 4}}{(\sqrt{|2 R o+1|})^{3 / 8}} .
$$

As is evident in figure 4(a,b), indeed, the most unstable mode's wavenumber decreases with increasing Ekman number, and increases with increasing stratification.

One can derive from this wavenumber selection the following scaling laws for the Rankine vortex: $m_{\max } \propto N^{1 / 4}$ and $m_{\max } \propto \operatorname{Re}^{3 / 8}$ (using $B u \propto N^{2}$ and the horizontal Reynolds number $\left.R e=V_{\max } r_{\max } / v \propto 1 / E k\right)$. These asymptotic results differ only slightly from the results in Kloosterziel et al. (2007), who found in a fully numerical nonlinear study for a family of barotropic vortices in a uniformly rotating and stratified fluid that the most unstable wavenumber in the nearly exponential growth stage follows $m_{\max } \propto N^{1 / 5}$ and $m_{\max } \propto R e^{1 / 3}$. The vertical scale selection for a parallel shear (derived in Kloosterziel \& Carnevale 2008) differs slightly more, and is $m_{\max } \propto N^{1 / 2}$ and $m_{\max } \propto R e^{1 / 4}$ for the linear case, and $m_{\max } \propto N^{1 / 3}$ and $m_{\max } \propto R e^{1 / 3}$ for a general parallel shear case with a non-vanishing second derivative of the absolute vorticity (derived in the Appendix by S. D. Griffiths in the same paper).

\subsection{Stability criterion for viscous and stratified Rankine vortices}

By equating the maximal growth in (3.10) to zero we find an analytic expression for the marginal stability curve:

$$
\frac{1}{E k}=\left[\frac{8\left|a_{0}\right|}{3}\right]^{3} B u \frac{|R o|^{2}}{(\sqrt{|2 R o+1|})^{7}} .
$$

In figure $3(b)$ this curve is compared to the one computed exactly by solving (3.8) with Mathematica software, for Burger numbers $B u=1,4$ and 36, with good 
agreement. We will see in what follows that this asymptotic marginal stability curve for the Rankine vortex can be extended to a wider range of non-isolated vortices. Therefore, (3.12) may be used as a generalized stability criteria for dissipative and stratified vortices.

As discussed in $\S 3.2,(3.12)$ shows that the critical $1 / E k$ decreases with increasing absolute value of the Rossby number $\left(1 / E k \propto R o^{-3 / 2}\right)$, and increases for increasing Burger number $(1 / E k \propto B u)$. As we also mentioned in $\$ 3.2$, similar behaviour was shown for a linear shear case by (Kloosterziel \& Carnevale 2008), who also developed an approximate analytic expression for the marginal stability curve (see equation (A 18)) for the strong stratification limit of this curve). It has the same dependence on the Burger number $(1 / E k \propto B u)$, but a different dependence on the Rossby number, and a different proportionality coefficient, that come from the adding the curvature (the centrifugal force) to the problem. It is interesting to note, however, that when the Rossby number is very large, the power law for both cases is $1 / E k \propto R o^{-3 / 2}$. Thus, the marginal stability curves for the linear shear and circular Rankine vortex (not shown) are similar for $R o \gg 1$ but very different for moderate vortex/shear intensities $(R o \sim O(1))$. The linear shear case approaches infinity for $R o \approx-1$, and the Rankine vortex approaches infinity only at $R o \approx-1 / 2$ (as in figure $3 a, b$ ).

\section{Stability of various vortices}

Among the wide variety of oceanic anticyclones there is no generic velocity profile. Previous studies on inertial instability of circular vortices (Gallaire \& Chomaz 2003; Kloosterziel et al. 2007) were devoted to isolated anticyclones. Such vortices have a negative vorticity core surrounded by a positive vorticity annulus and their circulation essentially vanishes at some final distance from the core. However, both the barotropic and the baroclinic instability of oceanic currents tend to form non-isolated vortices (with no changing sign of vorticity at the edge). Hence, we chose to compare the stability properties of various isolated and non-isolated vortices to the Rankine vortex. A non-isolated parabolic vorticity profile is given by

$$
\frac{\zeta(r)}{f}= \begin{cases}\frac{\zeta_{0}}{f}\left[1-\left(\frac{r}{R}\right)^{2}\right]=3 R o\left[1-\frac{2}{3} \eta^{2}\right] & r \leqslant R, \\ 0 & r \geqslant R,\end{cases}
$$

and a non-isolated conical vorticity profile by

$$
\frac{\zeta(r)}{f}= \begin{cases}\frac{\zeta_{0}}{f}\left[1-\frac{r}{R}\right]=4 R o\left[1-\frac{3}{4} \eta\right] & r \leqslant R, \\ 0 & r \geqslant R,\end{cases}
$$

where $R$ is the radius of the vorticity core, $\eta=r / r_{\max }$ and $r_{\max }$ the maximum velocity radius. The parabolic and conical vorticity profiles are relevant to vortices generated by coastal detachment, as shown in the accompanying paper on laboratory experiments on island wake vortices (Lazar et al. 2013). An isolated Rankine profile, with a ring of opposite sign vorticity between $R$ and $b R(b>1)$, is given by

$$
\frac{\zeta(r)}{f}= \begin{cases}\frac{\zeta_{0}}{f}=2 R o & r<R, \\ -\frac{\zeta_{0}}{f} \frac{1}{b^{2}-1}=2 R o \frac{1}{b^{2}-1} & R<r<b R, \\ 0 & r>b R,\end{cases}
$$




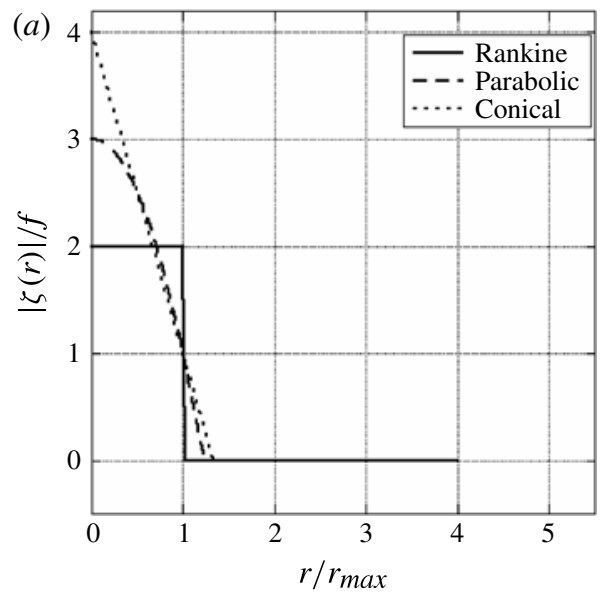

(b)

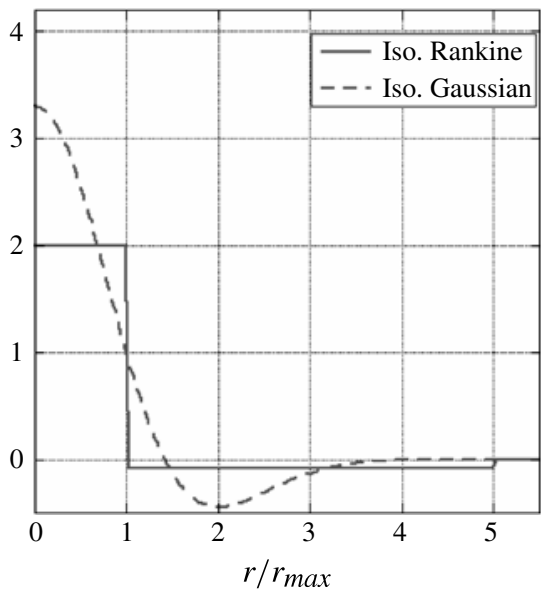

FIGURE 5. Vorticity profiles of ( $a$ ) non-isolated Rankine, parabolic and conical vortices, and $(b)$ isolated Rankine (with $b=5$ ) and Gaussian vortices. All profiles are for a Rossby number of $R o=-1$.

and an isolated Gaussian vortex by

$$
\frac{\zeta(r)}{f}=\frac{\zeta_{0}}{f} \mathrm{e}^{-\left(r^{2}\right) / 2}\left(1-\frac{r^{2}}{2}\right)=R o \mathrm{e}^{1 / 2-r^{2} / 2}\left(2-r^{2}\right) .
$$

The relation between relative core vorticity $\zeta_{0} / f$ and the vortex Rossby number $R o$ is very sensitive to the vortex type (vorticity and velocity profiles), and can be seen in the definition of the vorticity profiles (for the Rankine vortices it is $\zeta_{0} / f=2 R o$, for the parabolic vortex $\zeta_{0} / f=3 R o$, for the conical vortex $\zeta_{0} / f=4 R o$, and for the Gaussian vortex $\left.\zeta_{0} / f=2 R o \mathrm{e}^{-r^{2} / 2}\right)$. The vorticity profiles for these anticyclonic vortices with the same vortex Rossby number $R o=-1$ are given in figure 5(a,b). The normalized velocity profiles and the normalized Rayleigh discriminant are plotted in figure $6(a, b)$, respectively. Unlike the Rankine vortex where $R=r_{\max }$, the size of the vorticity core generally differs from the vortex radius and $R=\sqrt{3 / 2} r_{\max }\left(R=4 r_{\max } / 3\right)$ for the parabolic (conical) vortex. For both the isolated cases the vorticity core is $R=r_{\max }$ as well. Isolated vortices may also exhibit shear instabilities (Flierl 1988), so we choose $b=5$ in order for the isolated Rankine vortex to be stable to these.

As there is no direct analytical solutions for (2.20) when we consider vortex types other than Rankine, we analyse the stability of the different vortices by rewriting it as an eigenvalue problem, within the strong stratification regime (i.e. the hydrostatic case), which we will solve numerically:

$$
\left[\frac{B u}{(m \pi)^{2}}\left(\frac{\partial^{2}}{\partial \eta^{2}}+\frac{1}{\eta} \frac{\partial}{\partial \eta}-\frac{1}{\eta^{2}}\right)-\frac{\chi(\eta)}{f^{2}}\right] \phi_{n, m}=-\left(\frac{\omega_{\text {real }}}{f}+\mathrm{i} \hat{\sigma}\right)^{2} \phi_{n, m} .
$$

For every set of $B u, R o$ and $m$ (the Ekman number only enters the equation in the eigenvalue) we have $n$ discrete modes which are positive and a continuum spectrum of negative modes. These correspond to the $n$ growing/decaying standing waves $\left(\hat{\sigma} \neq 0\right.$ and $\left.\omega_{\text {real }}=0\right)$, each with 1 to $n$ horizontal nodes respectively, and the vertical propagating neutral waves $\left(\omega_{\text {real }} \neq 0\right.$ and $\left.\hat{\sigma}=0\right)$. As with the Rankine vortex, we concentrate only on the growing and decaying modes. 

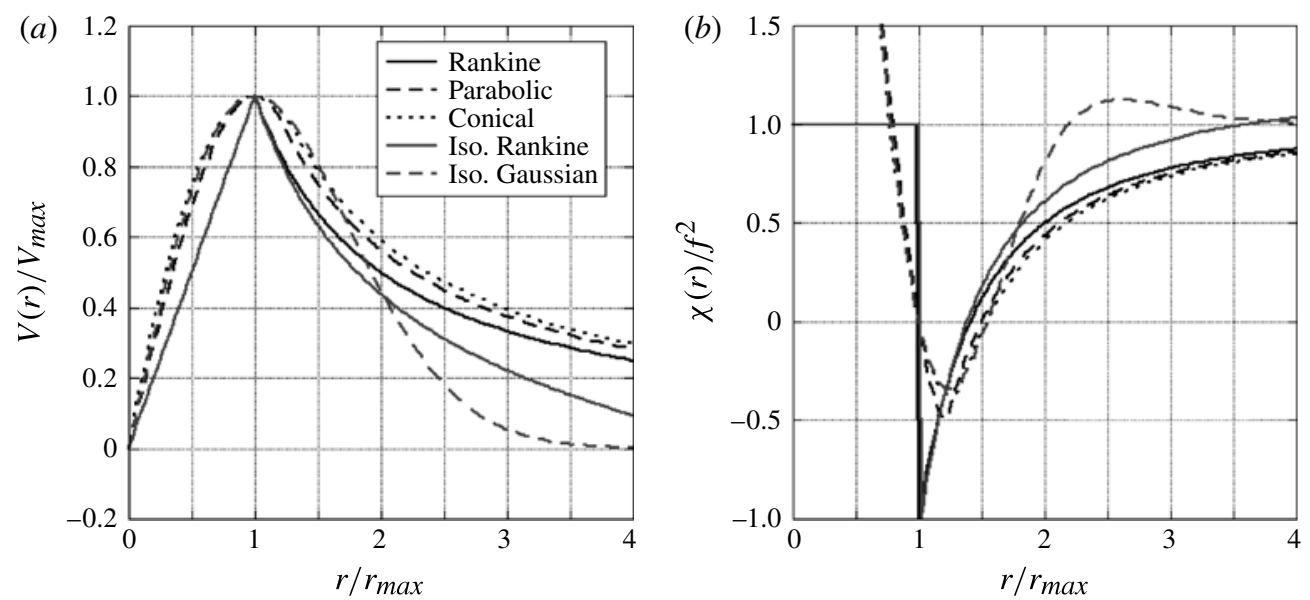

FIGURE 6. (a) Normalized velocity profiles of Rankine, parabolic, conical, isolated Rankine (with $b=5$ ) and isolated Gaussian vortices with a Rossby number of $R o=-1$. (b) The corresponding Rayleigh discriminants, $\chi(r)$.

\subsection{The vortex Rossby number: the relevant parameter}

As previously stated, the intensity of the vortex may be characterized by two different non-dimensional parameters: the vortex Rossby number $R o=V_{\max } / r_{\max } f$, similar to what is used in Kloosterziel et al. (2007), or the normalized core vorticity $\zeta_{0} / f$, as used, for instance, in Smyth \& McWilliams (1998). The ratio between them differs greatly for different vortex profiles, and it is not straightforward to decide a priori which one is more appropriate. We plot in figure $7(a, b)$ the marginal stability limits (i.e. $\sigma=0$ ) for the five types of vortices with $B u=4$. All these marginal curves coincide in figure 7(a), when drawn as a function of Ro. On the other hand, these stability curves differ greatly when drawn as a function of core vorticity (figure $7 b$ ). Similar results were found for other values of the Burger number (not shown). Further away from the marginal stability $R o$ is also a better parameter than $\zeta_{0} / f$, which is evident from figure $8(a, b)$. The growth rates of the unstable inertial modes are similar for anticyclones of the same value of $R o$.

Hence, as far as the inertial instability of circular viscid anticyclones is concerned, the vortex Rossby number appears to be the relevant parameter to quantify the vortex intensity. Furthermore, the idealized Rankine vortex is close to other types of vortices in its stability, meaning that all the analytical results obtained in $\S 3$, and especially in $\S 3.4$, can also be applied to the other vortices. Indeed, the analytical marginal stability curve (3.12), indicated in figure 7(a) by a grey solid line, coincides with the marginal stability curves of all the various vortices.

Nevertheless, for very weak dissipation, i.e. the inviscid limit (figure $7 a$ ), the marginal stability curves of the isolated and non-isolated Rankine vortices follow the analytic criterion well, whereas the other vortices diverge from it (in high values of $1 / E k)$. This is because in the inviscid limit one recovers the Rayleigh criterion. Note that for all vortices similar to those examined here (where the vorticity at the core is a global extremum), the Rayleigh criterion is equivalent to stating that a vortex is stable if the core absolute vorticity is positive (or in other words $\zeta_{0} / f>-1$ ). These inviscid limits for the different vortices are the vertical lines in figure $7(a)$. The fact that for very weak dissipation, the core vorticity, rather than the Rossby number, is 

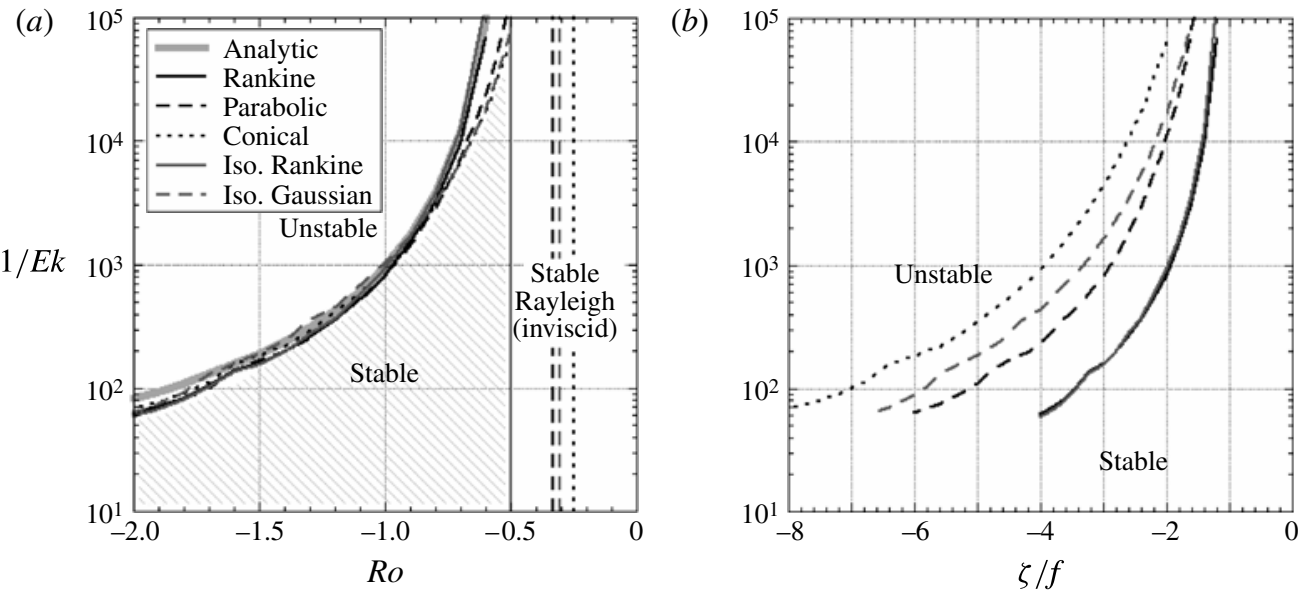

FIGURE 7. Marginal stability limit for the various types of vortices with stratification $(B u=4)$ as a function of $(a)$ the vortex Rossby number and $(b)$ the normalized vorticity. The curves are plotted only up to the supercritical limit and therefore stop at $R o=-\sqrt{B u}=-2$, and the respective $\zeta / f$. In $(a)$ the heavy solid grey line is the analytic criterion of (3.12), and the shaded area indicates the area that is predicted to be unstable by the Rayleigh criterion and is found to be stable by (3.12).
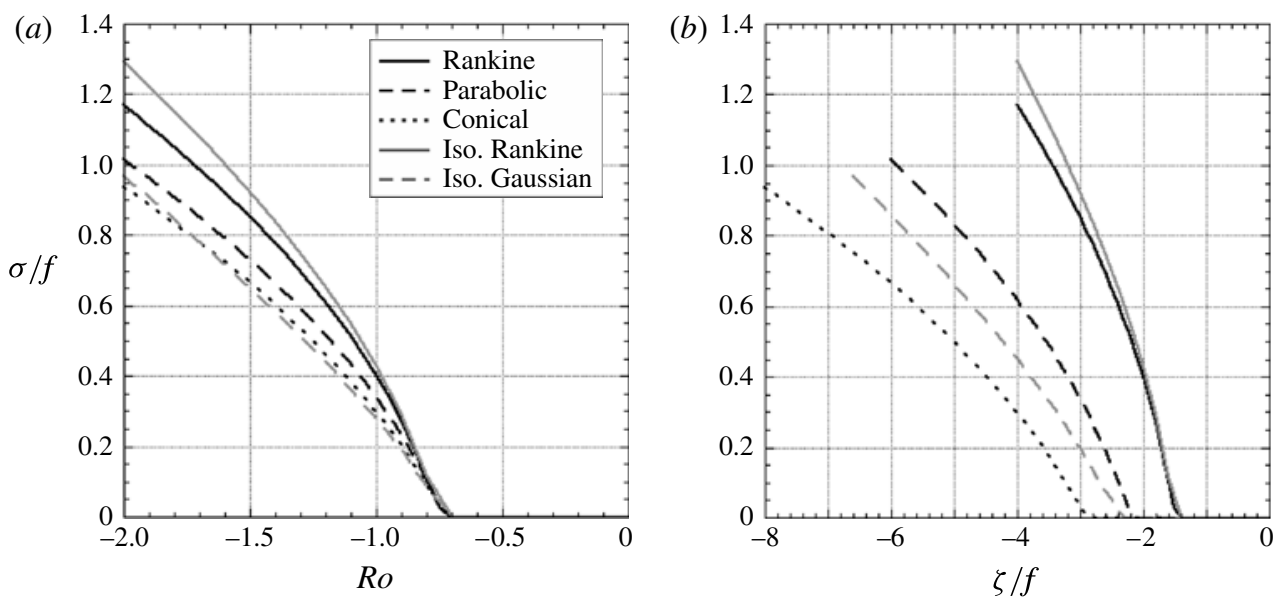

FIGURE 8. Growth rate for the various types of vortices, as a function of $(a)$ the vortex Rossby number and $(b)$ the normalized vorticity, in a stratified and viscid case $(B u=4, E k=1 / 5000)$. The curves are plotted only up to the supercritical limit.

the more suitable parameter is perhaps even more evident when looking at figure 9 . In this figure the analytical marginal stability limit (3.12) is delineated in the form of $\sqrt{B u}=R d / r_{\max }=F(R o, E k)$ as follows:

$$
\frac{R d}{r_{\max }}=\sqrt{B u}=\left[\frac{3}{8\left|a_{0}\right|}\right]^{3 / 2} \frac{1}{\sqrt{E k}} \frac{(|2 R o+1|)^{7 / 4}}{|R o|} .
$$

In figure 9 this curve is compared to the numerically computed marginal stability curves of the Rankine and parabolic vortices, for a number of Ekman numbers. The 


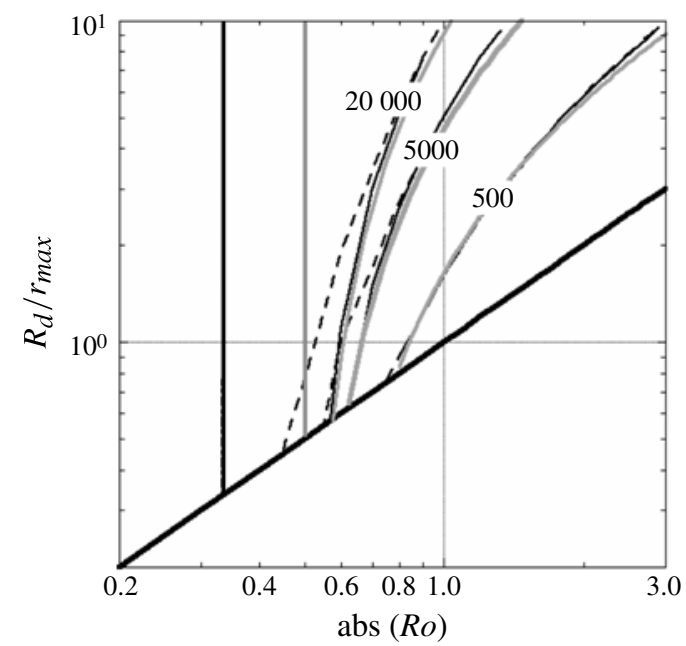

FIGURE 9. Comparison of marginal stability curves of the parabolic (black dashed) and Rankine (black solid) vortices, along with the analytic curve (grey solid) of several Ekman numbers on the $(\sqrt{B u}, R o)$ parameter space. The heavy solid line represents the supercritical limit. The dot-dashed line marks the inviscid stability limit for a parabolic vortex.

marginal stability curves of the analytical and numerical Rankine stability curves fit together well. However, we find significant differences between the marginal stability curves between the parabolic and the (analytical and numerical) Rankine vortex for $1 / E k=2 \times 10^{4}$. In this figure it is clear that the difference stems from the fact that in weak dissipation the two different types of vortices tend to a different vortex Rossby number, which corresponds to $\zeta_{0} / f=-1$ : the Rankine vortices to $R o=-1 / 2$; and the parabolic to $R o=-1 / 3$. To summarize, (3.12) and (4.6) can be used as a general criterion for various vortices to replace the inviscid Rayleigh criterion when the vertical dissipation is not too small.

\subsection{Mapping of the parameter space}

For a given thickness, $h$, the Ekman number, $E k$, is fixed. The inertial instability is then driven by the vortex Rossby number and the relative vortex size in comparison with the local deformation radius. We plot in figure 10(a) the growth rate amplitudes of the most unstable axisymmetric inertial modes in the $\left(R d / r_{\max }=\sqrt{B u}, R o\right)$ parameter space for a parabolic vortex when $1 / E k=5000$, and in figure $10(b)$ we plot the vertical wavenumber corresponding to this mode. Anticyclonic structures become unstable when the vortex Rossby number reaches finite negative values. In order to maintain a subcritical Froude number (the heavy solid line in the figure), these eddies should also be small in comparison with the deformation radius $\left(r_{\max }<R d\right)$. However, if the eddy radius decreases while the Rossby number is kept fixed, the inertial growth rates decreases. Hence, if the eddies are too small, they will not be strongly affected by the inertial perturbations. Therefore, as shown in figure 10(a), the most unstable anticyclones are the submesoscale eddies which are close to the subcritical limit. These are also the largest vertical modes, as is evident from figure $10(b)$, where we see that the vertical modes mostly depend on the Burger number. Notice that the unstable region predicted by (4.6) is in good agreement with the region of positive growth rates in figure $10(a)$, calculated numerically for the parabolic vortex. 

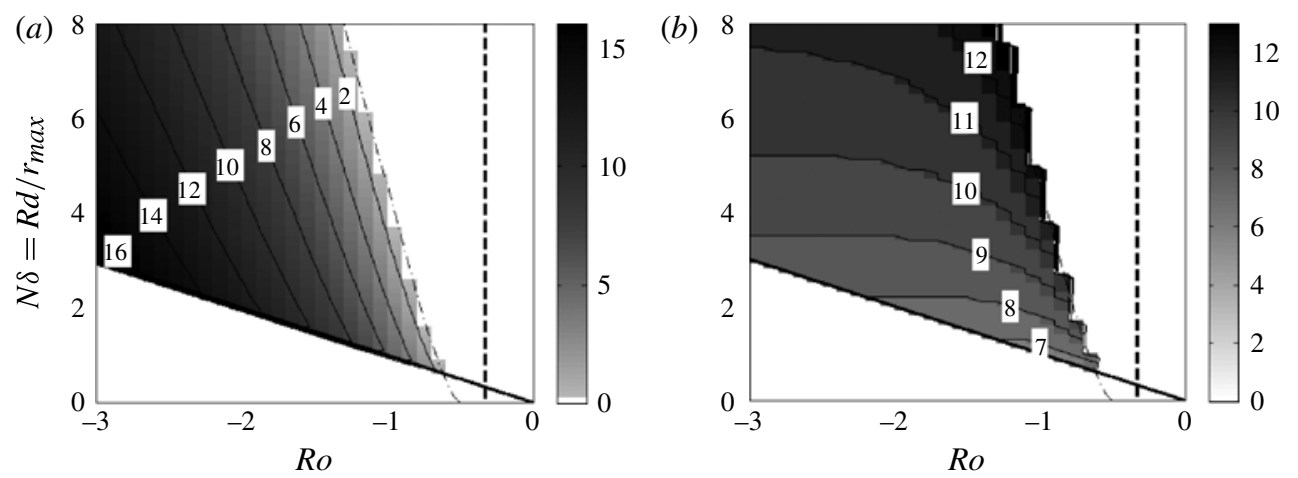

FIGURE 10. (a) Map of the growth rate, $\sigma T$, of the most unstable axisymmetric inertial modes in the $\left(R d / r_{\max }=\sqrt{B u}, R o\right)$ parameter space for a parabolic vortex, with $E k^{-1}=5000$. The dot-dashed line is the analytic marginal stability curve in (4.6). (b) The corresponding vertical wavenumber, $m$. In both panels the thick solid line is the $F r=1$ line, beneath which the fluid is supercritical. The broken line is the inviscid limit, $R o>-1 / 3$, to the right of which the Rayleigh discriminant is positive everywhere in the flow.

\section{Summary and discussion}

We have investigated the stability of various submesoscale circular vortices to three-dimensional inertial perturbations. The main purpose of this work was to build a stability diagram and achieve a stability criterion more suitable for geophysical vortices, where stratification and dissipation both play vital roles. We first performed a linear stability analysis of the classical barotropic Rankine vortex in a rotating and linearly stratified shallow and viscous fluid layer. For this idealized vortex the stability of axisymmetric inertial perturbations was solved by means of asymptotic expansion, and we obtained an analytic dispersion relation from which we found the expected maximal growth rate for any set of parameters. Furthermore, we derived for the Rankine vortex a marginal stability limit equation which depends only on three dimensionless parameters: the vortex Rossby number, $R o$, the Burger number, $B u$, and the Ekman number, $E k$. This criterion is the natural 'next step' from the generalized Rayleigh criterion which is valid only for non-dissipative and non-stratified eddies.

Our stability analysis has shown that a strong stratification enhances the impact of dissipation, making the Ekman number a crucial parameter for the centrifugal-inertial instability. Quite surprisingly, we find that intense anticyclones could be stable even if they have a core region of negative absolute vorticity (i.e. $\zeta(0)<-f$ ), provided they are small enough. Indeed, for given Rossby and Ekman numbers, the growth rate of inertial perturbations, within a circular vortex, decays when the Burger number increases. In the hydrostatic regime (i.e. strong stratification), which is equivalent to high Burger numbers (i.e. large $R d \propto N$ in comparison with eddy radius $r_{\max }$ ), the vertical scale of the unstable perturbations will be damped by the viscous dissipation. Our stability diagram (figure 10) shows that to be unstable a strongly stratified anticyclone should have a sufficiently negative vortex Rossby number and the most unstable ones are close to the supercritical limit $\left(R d / r_{\max }=R o\right)$.

The marginal stability of a linear shear (also taking into account stratification and dissipation, as in Kloosterziel \& Carnevale 2008) is different from that found here for the Rankine vortex. For moderate Rossby numbers, the linear shear is more stable 
than the circular. This makes sense, because in the circular case the centrifugal force is acting together with the pressure gradient force against the Coriolis force. The curves are only similar when $R o \gg 1$. Then, the pressure gradient force is so strong that a factor of two (approximately), coming from the centrifugal force, is not very influential.

Next, we found that if one uses the vortex Rossby number (as opposed to the normalized core relative vorticity, which is often used for this purpose) the marginal stability curves for various types of stratified and viscous vortices (non-isolated Rankine, parabolic, conical and also isolated Gaussian and Rankine vortices) collapse into a single curve. This means that the asymptotic marginal stability limit, derived for a Rankine vortex and the stability diagram plotted in the $(R o, \sqrt{B u}, E k)$ parameter space, is applicable to a wide variety of vortices. Hence, (3.12) and (4.6) could be used as a generalized stability limit for viscid vortices embedded in a stratified layer (as noted, in the inviscid limit the Rayleigh criterion is recovered, and the core vorticity is the better parameter).

In geophysical vortices, where stratification and dissipation are both important, this analysis is better suited to explaining the stability. This is exactly the case, for instance, in large-scale laboratory experiments that mimic island wakes (Teinturier et al. 2010, and our accompanying paper, Lazar et al. 2013), where the inverse Ekman number is moderate $\left(1 / E_{k}=300-600\right)$ and the vertical dissipation cannot be neglected. This is also the case when considering oceanic vortices. The aspect ratio is usually small, $\delta \sim 10^{-1}-10^{-2}$, and the stratification is strong. Note that the barotropic idealization, which neglects the isopycnal tilting and corresponding vertical shear (or equivalently the divergent dynamics of a two-layer shallow water model), is a strong simplification for geophysical vortices. When the mean flow velocity exhibits a vertical shear, symmetric instability can develop, which expands the unstable region in the mesoscale area (see appendix C). Therefore, large mesoscale eddies (larger than the deformation radius) may exhibit symmetric instability. Nonetheless, this is unlikely to change the limit for submesoscale eddies, hence when the Burger number is larger than unity the new criterion holds.

In the real ocean, estimating the Burger number and the vortex intensity can be quite simple: a single density profile and the velocity field give the information needed, even more so in this case, since a much lower resolution is required to estimate the Rossby number (which our analysis shows is a more suitable parameter for the ocean) than is required to estimate the normalized vorticity. However, estimating the Ekman number is not straightforward, as we should consider the turbulent diapycnal mixing (i.e. the vertical turbulent eddy viscosity) instead of the molecular viscosity. Assuming that intense submesoscale eddies are generally formed at the ocean surface by strong shear flows (like in the lee of islands) or significant wind stress curl, we could use, as a first guess, a large diapycnal diffusivity of $\kappa_{z} \sim v_{z} \sim 10^{-4} \mathrm{~m}^{2} \mathrm{~s}^{-1}$, keeping in mind that it may vary by an order of magnitude. The corresponding Ekman number will then vary with the thermocline depth and the latitude. For a typical vortex depth of $h \sim 100 \mathrm{~m}$ we will get $1 / E k \sim 10 \times 10^{3}$ at mid-latitudes and $1 / E k \sim 3 \times 10^{3}$ in equatorial regions. Maximal velocities of $0.3-0.6 \mathrm{~m} \mathrm{~s}^{-1}$ and radii of $5-15 \mathrm{~km}$, which are typical values seen in island wake vortices, correspond to $B u>1$ and $R o=0.4-0.6$ in the mid-latitudes. Hence, it seems that our stability diagram (figure 9) is relevant to the stability of oceanic vortices, since even for $1 / E k$ as high as $20 \times 10^{3}$ these vortices should be stable, regardless of their specific vorticity profile, even if $\zeta_{0} / f<-1$. This is contrary to the Rayleigh criterion, which corresponds to the black (grey) vertical lines 


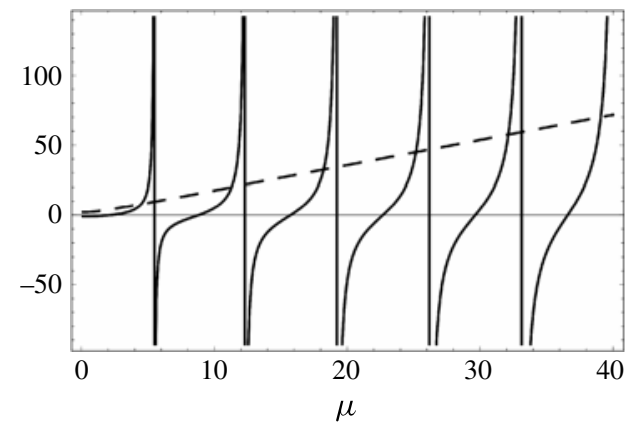

FIGURE 11. The left-hand and right-hand sides of (3.8) plotted as a function of $\mu=-\mathrm{i} v$ (dashed line and solid line, respectively).

in figure 9 for the parabolic (Rankine) vortex. However, a remaining issue in applying this theory to the ocean is to have a clear signature that can help identify that an eddy is going through inertial instability. In our accompanying paper (Lazar et al. 2013) we find such a signature that is sensitive enough to detect the instability even in its initial stage.

\section{Acknowledgements}

Ayah Lazar and Eyal Heifetz would like to thank the Israeli Ministry of Science and Technology (MOST 3-6490) for funding this research. Ayah Lazar also acknowledges the sustainable development chair of the École Polytechnique for his support. Eyal Heifetz is grateful to the International Meteorological Institute (IMI) of Stockholm University for hosting him in the Meteorological Institute of Stockholm University (MISU). Alexandre Stegner would like to thank the French ANR-Flowing project (part of the ANR 'Programme Blanc') for funding this research. We are all very grateful to Riwal Plougonven and Paul Billant for the enlightening discussions.

\section{Appendix A. Analytic solution for the Rankine vortex}

In this appendix we will develop an asymptotic dispersion relation, $\sigma / f=$ $F(R o, B u, E k ; m)$. According to (3.3), inside the Rankine vortex the equation is a modified Bessel function of order one, and outside the vortex it is a modified Bessel function of order $\gamma$. Taking into account the radial boundary conditions, the solution is the piecewise eigenmode given in (3.7), which with the requirement of continuity gives the implicit equation (3.8). This equation may be solved exactly using the Mathematica solver. A plot of the right-hand side and the left-hand side of the equation as a function of $\mu$ (the real factor of the order of the Bessel function $\gamma \equiv \mathrm{i} \mu$, and $\mu \in \mathrm{Re}$ ), reveals that they intersect roughly where $\mathrm{K}_{\mathrm{i} \mu}(\beta)=0$ (see figure 11 ). This is because the left-hand side is a steadily increasing function, whereas the right-hand side periodically goes to infinity very quickly, and as $\mathrm{K}_{\mathrm{i} \mu}$ and $\mathrm{K}_{\mathrm{i} \mu}^{\prime}$ are bounded functions (except in the origin), this can only happen if $\mathrm{K}_{\mathrm{i} \mu}=0$, from (3.8).

Under this assumption we will find an analytical term for the dispersion relation $(\sigma(m))$, by solving

$$
\mathrm{K}_{\mathrm{i} \mu(R o, B u ; \hat{\sigma}, m)}(\beta(R o, B u ; \hat{\sigma}, m))=0,
$$


where $\gamma($ or $\mu$ ) and $\beta$ in terms of $R o, B u, \hat{\sigma}$ and $m$ (in the hydrostatic approximation, for simplicity) are

$$
\begin{array}{r}
\mu=-\mathrm{i} \gamma \approx \sqrt{-\left(1+\frac{(m \pi)^{2}}{B u} 2 R o\right)}, \\
\beta \approx \sqrt{\frac{(m \pi)^{2}}{B u}\left(1+\hat{\sigma}^{2}\right)}=\sqrt{-\frac{\mu^{2}+1}{2 R o}\left(1+\hat{\sigma}^{2}\right)} .
\end{array}
$$

The solutions of (A 1$)$ are $\beta=k_{\mu, s}$, where $k_{\mu, s}$ is the sth zero of the $\mathrm{K}_{\mathrm{i} \mu}$. It has been shown (Dunster 1990) that $\mathrm{K}_{\mathrm{i} \mu}$ has an infinite number of zeros for $0<\beta<\mu$, and none for $\mu<\beta<\infty$. This means that for there to be any solution $\beta$ has to be smaller than $\mu$, and as they are both real, we can say that $\beta^{2}<\mu^{2}$, which gives

$$
\hat{\sigma}<\sqrt{-(2 R o+1)-\frac{B u}{(m \pi)^{2}}}=\sqrt{|\chi|_{\max }-\frac{B u}{(m \pi)^{2}}} .
$$

This is close to the inviscid maximum growth we obtained earlier $\left(\hat{\sigma}<\sqrt{|\chi|_{\max }}\right)$, but gives us a slightly stricter limit, depending on the stratification (when $m$ is large they grow closer).

The zeros of $\mathrm{K}_{\mathrm{i} \mu}$ for large $\mu$ (which is the large $m$ regime) are given by

$$
k_{\mu, s}=\mu+a_{s}\left(\frac{\mu}{2}\right)^{1 / 3}+\frac{3}{20} a_{s}^{2}\left(\frac{\mu}{2}\right)^{-1 / 3}+O\left(\mu^{-2 / 3}\right),
$$

where $a_{s}$ is the sth zero of the Airy function, which are all negative and satisfy $0<a_{0}<a_{1}<\cdots<a_{s}$. With this (A 1 ) can be rewritten as

$$
\beta=\sqrt{-\frac{\mu^{2}+1}{2 R o}\left(1+\hat{\sigma}^{2}\right)}=\mu+a_{s}\left(\frac{\mu}{2}\right)^{1 / 3}+\frac{3}{20} a_{s}^{2}\left(\frac{\mu}{2}\right)^{-1 / 3}+O\left(\mu^{-2 / 3}\right) .
$$

Assuming $\mu \geqslant 1$ (true if $m^{2} \geqslant B u /\left(\pi^{2}|R o|\right.$ ), which is very probable given that $B u /|R o| \sim O(1)$, and we have assumed that we are in the large $m$ regime), solving for $\hat{\sigma}^{2}$ gives

$$
\begin{aligned}
\hat{\sigma}^{2} & =-1-\frac{2 R o}{\mu^{2}+1}\left(\mu+a_{s}\left(\frac{\mu}{2}\right)^{1 / 3}+\frac{3}{20} a_{s}^{2}\left(\frac{\mu}{2}\right)^{-1 / 3}+O\left(\mu^{-2 / 3}\right)\right)^{2} \\
& =-1-\frac{2 R o}{\mu^{2}+1}\left[\mu^{2}+2 \frac{a_{s}}{2^{1 / 3}} \mu^{4 / 3}\right]+O\left(\mu^{2 / 3}\right) .
\end{aligned}
$$

Notice that for the first order in $\mu$ we get $\hat{\sigma}^{2}=-1-2 R o / \mu^{2}+1 \mu^{2}$, which is the condition to have at least one solution $(\beta=\mu)$. Considering the next order, plugging in $\mu$ and expanding for large $m$ we have

$$
\hat{\sigma}^{2}=-(2 R o+1)-\frac{B u}{(m \pi)^{2}}+2^{2 / 3} a_{s}\left(\frac{B u}{(m \pi)^{2}}\right)^{1 / 3}(-2 R o)^{2 / 3}+O\left(\mu^{-10 / 3}\right) .
$$

Expanding again we get

$$
\hat{\sigma} \approx \sqrt{-(2 R o+1)}\left(1+a_{s} B u^{1 / 3} \frac{(-\sqrt{2} R o)^{2 / 3}}{-(2 R o+1)}(\pi m)^{-2 / 3}\right) .
$$


We note that if we substitute $|\chi|_{\max }=1+2 R o$, and the derivative $\chi_{\max }^{\prime}=-4 R o$, we get

$$
\hat{\sigma} \approx \sqrt{-\chi_{\max }}+a_{s} \frac{\chi_{\max }^{\prime 2 / 3}}{2 \sqrt{-\chi_{\max }}}\left(\frac{B u}{(m \pi)^{2}}\right)^{1 / 3} .
$$

This is very similar to what we get by adapting the WKB approximation in Billant \& Gallaire (2005) for a Taylor-Couette flow to the stratified case:

$$
\hat{\sigma} \approx \sqrt{-\chi_{\max }}-\frac{1}{8 m^{2 / 3} \sqrt{-\chi_{\max }}}\left(9 \pi \chi_{\max }^{\prime} \sqrt{-\chi_{\max }}\right)^{2 / 3}\left(1+\frac{B u}{-\chi_{\max }}\right)^{1 / 3} .
$$

\section{A.1. Growth rate}

Finally, taking into account the viscosity, $\sigma / f=\hat{\sigma}-(m \pi)^{2} E k$, we have the dispersion relation (remembering that $\omega_{\text {real }}=0$ )

$$
\frac{\sigma}{f} \approx \sqrt{|2 R o+1|}\left(1+a_{s}\left(\frac{\sqrt{2}}{\pi}\right)^{2 / 3} \frac{|R o|^{2 / 3}}{|2 R o+1|} B u^{1 / 3} m^{-2 / 3}\right)-(m \pi)^{2} E k,
$$

so that the leading order is $m^{2}$, and at large $m$ s the growth rate decays like $\propto-m^{2}$. For a given set of parameters, the growth rate decreases for higher zeros (the subscript $s$ ) of the Airy function, $a_{s}$, which correspond to a higher radial mode $n$.

\section{A.2. Marginal stability}

The marginal stability curve $\left(E k^{-1}=F(R o, B u)\right)$ is found by equating to zero the maximal growth rate. We differentiate (A 12) by $m$ and equate to zero to find $m_{\max }$ :

$$
\left.\frac{1}{f} \frac{\partial \sigma}{\partial m}\right|_{m=m_{\max }}=-2 m\left(\frac{1}{3} a_{0}\left(\frac{\sqrt{2}}{\pi}\right)^{2 / 3} \frac{|R o|^{2 / 3}}{\sqrt{|2 R o+1|}} B u^{1 / 3} m^{-8 / 3}+\pi^{2} E k\right)=0, \text { (A 13) }
$$

where we have taken $a_{0}=-2.33811$ as we are looking for the most unstable mode, and the first radial mode is always the largest. Thus, the vertical mode that corresponds to the maximal growth rate is

$$
m_{\max }=\frac{2^{1 / 8}\left|a_{0}\right|^{3 / 8}}{3^{3 / 8} \pi} B u^{1 / 8} E k^{-3 / 8} \frac{|R o|^{1 / 4}}{(\sqrt{|2 R o+1|})^{3 / 8}},
$$

as $m=0$ is not a valid solution with the top and bottom boundary conditions (except the trivial solution of $\phi_{n, 0}=0$ ).

The maximal growth is

$$
\left(\frac{\sigma}{f}\right)_{\max } \approx \sqrt{|2 R o+1|}\left(1-(B u E k)^{1 / 4} \frac{|R o|^{1 / 2}}{(\sqrt{|2 R o+1|})^{7 / 4}}\left[\frac{8\left|a_{0}\right|}{3}\right]^{3 / 4}\right) .
$$

For marginal stability, we equate this to zero and get the curve

$$
\frac{1}{E k}=B u \frac{|R o|^{2}}{(\sqrt{|2 R o+1|})^{7}}\left[\frac{8\left|a_{0}\right|}{3}\right]^{3} \text {. }
$$

We shall compare the above marginal stability curve of the Rankine vortex to that derived by Kloosterziel \& Carnevale (2008) for the case of a linear 
shear $\left(U(y)=U_{0}+\Lambda y\right)$, which also takes into account the vertical eddy viscosity and constant stratification. Assuming strong stratification, in order to compare with our results which are in the hydrostatic approximation, their equation (4.9) for the maximal growth rate becomes

$$
s_{*}=|f Q|^{1 / 2}\left[1-\left(2 \pi^{2}\right)^{1 / 2} \frac{N}{|f Q|^{3 / 4}} \frac{(|f Q|+1)^{1 / 2}}{R e^{1 / 2}}\right] .
$$

Equating this to zero, and translating it into the terms of (A 16), gives us the marginal stability curve

$$
\frac{1}{E k}=2 \pi^{2} B u \frac{1}{(|R o+1|)^{3 / 2}},
$$

where we have used $1 / E k=R e \delta^{2} / R o, B u=N^{2} \delta^{2}, R o=-\Lambda / f$ (the minus sign is added to have the same convention, with anticyclonic shear being negative Rossby number), and $|f Q|=f^{2}|1+R o|$. It is important to note that the comparison of these marginal stability curves as a function of the Rossby numbers requires us to be careful to define it in a consistent way. We find in this paper that the relevant parameter for comparing the circular cases is $R o=V_{\max } / r_{\max } f$ (see $\S 4.1$ ), so we would like to compare the parallel shears in the same way. Assuming the shear is symmetric about the $x$-axis, $V_{\max }$ is the maximal velocity that is reached at distance $r_{\max }$. For a linear shear this is the same as the core vorticity, but for other types of shear this would be different.

\section{Appendix B. Vertical wavenumber range}

The range of allowed vertical wavenumbers, $m_{B u}<m<m_{E k}$, can be understood from analytical formulae if we note that in order for the solutions of (3.8) inside and outside to fit together continuously and smoothly (continuous $\phi$ and $\phi^{\prime}$ ), the order, $\gamma$, should be imaginary:

$$
\gamma^{2}=1+(m \pi)^{2} \frac{2 R o}{B u+H y \delta^{2} \hat{\sigma}^{2}}<0 .
$$

Remembering that $\hat{\sigma}=\sigma / f+(m \pi)^{2} E k$, we get a quadratic equation for $m^{2}$,

$$
H y E k^{2} \delta^{2} \pi^{4} m^{4}+2 \pi^{2}\left(H y \delta^{2} \frac{\sigma}{f} E k+R o\right) m^{2}+B u+H y \delta^{2}\left(\frac{\sigma}{f}\right)^{2}<0,
$$

which implies a short and large wavelength cutoff, $m_{\text {lower }}<m<m_{\text {upper }}$, for the case of marginal stability $(\sigma / f=0)$. Notice that this condition is weak, and when checking $\gamma^{2}$ for the whole range of $m$ where $\sigma / f \geqslant 0$, it is always smaller than zero $\left(\gamma^{2}<0\right)$, so that the actual range of $m$ is smaller $\left(m_{\text {lower }}<m_{B u}<m<m_{E k}<m_{\text {upper }}\right)$. For marginal stability this equation becomes

$$
H y E k^{2} \delta^{2} \pi^{4} m^{4}+2 R o(\pi m)^{2}+B u<0,
$$

For strong stratification the hydrostatic approximation $(H y=0)$ is satisfied automatically, and this equation becomes

$$
2 R o(\pi m)^{2}+B u<0 .
$$


Remembering that we are focusing on anticyclones $(R o<0)$, and that $m>0$, this gives

$$
m>m_{\text {lower }}=\frac{1}{\pi} \sqrt{\frac{B u}{-2 R o}},
$$

which shows that the lower cutoff is indeed proportional to the stratification.

The short wavelengths are cut off by the dissipation. We examine this by looking at the non-hydrostatic case $(H y=1)$, for which the solutions are

$$
\left(m_{1,2}\right)^{2}=\frac{-R o \pm \sqrt{R o^{2}-E k^{2} \delta^{2} B u}}{\pi^{2} E k^{2} \delta^{2}}=\frac{|R o|}{\pi^{2} E k^{2} \delta^{2}}\left(1 \pm \sqrt{1-\frac{E k^{2} \delta^{2} B u}{R o^{2}}}\right) .
$$

Looking at the unstratified case, for simplicity, we get

$$
m<m_{\text {upper }}=\frac{\sqrt{2|R o|}}{\pi \delta E k},
$$

showing that the upper cutoff is indeed induced by the dissipation. Looking at the general case,

$$
\left(m_{\text {upper }}\right)^{2}=\frac{|R o|}{\pi^{2} E k^{2} \delta^{2}}\left(1+\sqrt{1-\frac{E k^{2} \delta^{2} B u}{R o^{2}}}\right),
$$

it is evident that stratification reduces this upper limit.

\section{Appendix C. Limitations of the analysis}

As noted in $\S 5$, the barotropic idealization, which neglects the isopycnal tilting, is a strong simplification for real geophysical vortices. When the mean flow is baroclinic (either continuously or in a two-layer reduced gravity model) symmetric instability can develop. The symmetric instability has been widely studied for parallel flows and/or fronts (Stone 1966; Haine \& Marshall 1998; Holton 2004; Taylor \& Ferrari 2009), and for inviscid circular flows with swirl velocity in a stably stratified fluid (Sawyer 1947; Ooyama 1966; Charney 1973). Unstratified, viscous circular vortices were studied by Kloosterziel (2010), where the absolute vorticity is non-zero everywhere in the flow, but even then, only a Lyapunov stability analysis is possible. There is no complete study on symmetrical instability of circular baroclinic vortices taking into account both the stratification and the dissipation, and the extension of the stability criteria cited above to this case is not direct. For an inviscid stably stratified circular baroclinic vortex we can use the aforementioned studies, namely the negative potential vorticity (PV) criterion for symmetric instability. The Ertel PV for a Boussinesq fluid is $\Pi=\left(f \boldsymbol{e}_{z}+\nabla \times \boldsymbol{u}\right) \cdot \nabla \boldsymbol{b}$, where $b=-g \rho(r, z) / \rho_{0}$. For the mean flow of an axisymmetric vortex satisfying the hydrostatic approximation, we have the cyclogeostrophic and thermal wind balance equation for the azimuthal velocity $V(r, z)$ :

$$
\left(\frac{2 V}{r}+f\right) \partial_{z} V=-\frac{g}{\rho_{0}} \partial_{r} \rho .
$$

The Ertel PV, using this equation, is

$$
\Pi=(f+\zeta) N^{2}-\left(\frac{2 V}{r}+f\right)\left(\frac{\partial V}{\partial z}\right)^{2},
$$




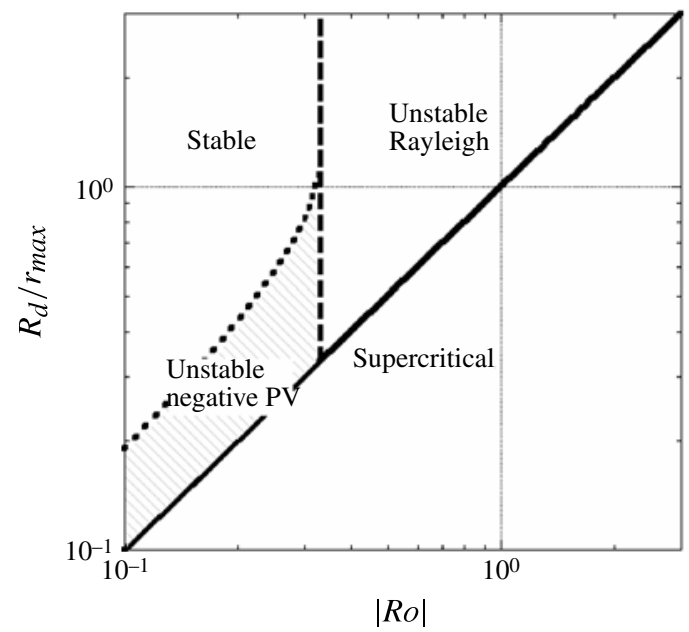

FIGURE 12. The marginal stability limits according to the Rayleigh criterion (dashed line) for a barotropic parabolic vortex, and according to the negative PV criterion (dotted line) for a baroclinic parabolic vortex on the $(\sqrt{B u}, R o)$ parameter space. The heavy solid line represents the supercritical limit, and the shaded area indicates where only symmetrical modes are unstable.

where $\zeta=1 / r \partial_{r}(r V)$ is the vertical component of the mean flow's vorticity, and $N^{2}=-g / \rho_{0} \partial_{z} \rho$ is the Brunt-Väisälä frequency, which we assume is constant. Considering a linear vertical velocity shear $V(r, z)=\tilde{V}(r) z / h$, we get

$$
\Pi=\left(f+\tilde{\zeta} \frac{z}{h}\right) N^{2}-\left(\frac{2 \tilde{V}}{r} \frac{z}{h}+f\right)\left(\frac{\tilde{V}}{h}\right)^{2},
$$

where $\zeta=1 / r \partial_{r}(r \tilde{V})$. Using the dimensionless formulation $\eta=r / r_{\max }$ and $\bar{V}=v / V_{\max }$, and dividing by $f^{2} N^{2}$ we get the following condition for negative PV:

$$
\left(1+\frac{\tilde{\zeta}}{f} \frac{z}{h}\right)-F r^{2}\left(1+2 \operatorname{Ro} \frac{z}{h} \frac{v}{\eta}\right)\left(\frac{v}{\tilde{z}}\right)^{2}<0,
$$

where the Rossby and Froude numbers are defined as before, i.e. $R o=V_{\max } /\left(r_{\max } f\right)$ and $F r=R o^{2} / B u=V_{\max }^{2} /(N h)^{2}$. If the Froude number is subcritical the region of negative PV will be close to the free surface. Hence, the symmetric instability should first appear at $z=h$, and we look at the criterion for this case.

If we plug in the velocity structure of a parabolic vorticity profile from $\S 4$, we get

$$
\begin{cases}\left(1+3 \operatorname{Ro}\left(1-\frac{2}{3} \eta^{2}\right)\right), & \\ -\left(1+3 \operatorname{Ro}\left(1-\frac{\eta^{2}}{3}\right)\right)^{2} \frac{9}{4} \eta^{2}\left(1-\frac{\eta^{2}}{3}\right) F r^{2}<0, & \eta<\sqrt{3 / 2} \\ 1-\left(1+\frac{9}{4} \operatorname{Ro} \frac{1}{\eta^{2}}\right)^{2}\left(\frac{9}{8 \eta}\right) F r^{2}<0, & \eta \geqslant \sqrt{3 / 2} .\end{cases}
$$

The marginal stability (negative PV) line is found numerically, and is indicated in figure 12 by a dotted line. The gap between this marginal stability curve and the one 
for inertial instability (the shaded area) indicates the area that corresponds only to the symmetrical instability, due to tilted isopycnals. According to the inviscid case, we can estimate that the isopycnal titling (i.e. the symmetric instability) may have an impact on the marginal stability curve when the Burger number is below unity, in other words for large mesoscale vortices close to the supercritical limit. Hence, our stability criterion is fully relevant to submesoscale $(B u>1)$ vortices within the thermocline or the mixed layer.

\section{REFERENCES}

AfANASYEV, Y. D. 2002 Experiments on instability of columnar vortex pairs in rotating fluid. Geophys. Astrophys. Fluid Dyn. 96 (1), 31-48.

Afanasyev, Y. D. \& Peltier, W. R. 1998 Three-dimensional instability of anticyclonic swirling flow in rotating fluid: laboratory experiments and related theoretical predictions. Phys. Fluids 10 (12), 3194-3202.

Bartello, P., Métais, O. \& Lesieur, M. 1994 Coherent structures in rotating three-dimensional turbulence. J. Fluid Mech. 273 (1), 1-29.

Billant, P. \& Gallaire, F. 2005 Generalized Rayleigh criterion for non-axisymmetric centrifugal instabilities. J. Fluid Mech. 542, 365-379.

Carnevale, G. F., Briscolin, M., Kloosterziel, R. C. \& Vallis, G. K. 1997 Three-dimensionally perturbed vortex tubes in a rotating flow. J. Fluid Mech. 341 (1), $127-163$.

Carnevale, G. F., Kloosterziel, R. C., Orlandi, P. \& van Sommeren, D. D. J. A. 2011 Predicting the aftermath of vortex breakup in rotating flow. J. Fluid Mech. 669, 90-119.

Charney, J. G. 1973 Planetary fluid dynamics. In Dynamic Meteorology (ed. P. Morel), pp. 97-351. Springer.

Chavanne, C., Flament, P. \& Gurgel, K.-W. 2010 Interactions between a submesoscale anticyclonic vortex and a front. J. Phys. Oceanogr. 40 (8), 1802-1818.

DUNSTER, T. M. 1990 Bessel functions of purely imaginary order, with an application to second-order linear differential equations having a large parameter. SIAM J. Math. Anal. 21 (4), 995-1018.

Flierl, G. R. 1988 On the instability of geostrophic vortices. J. Fluid Mech. 197, 349-388.

Gallaire, F. \& Chomaz, J.-M. 2003 Three-dimensional instability of isolated vortices. Phys. Fluids 15 (8), 2113-2126.

GRIFFITHS, S. D. 2008 The limiting form of inertial instability in geophysical flows. J. Fluid Mech. 605, 115-143.

Haine, T. W. N. \& Marshall, J 1998 Gravitational, symmetric, and baroclinic instability of the ocean mixed layer. J. Phys. Oceanogr. 28, 634-658.

Holton, J. R. 2004 An Introduction to Dynamic Meteorology. Academic Press.

Johnson, J. A. 1963 The stability of shearing motion in a rotating fluid. J. Fluid Mech 17 (3), 337-352.

Kloosterziel, R. C. 2010 Viscous symmetric stability of circular flows. J. Fluid Mech. 652, 171-193.

Kloosterziel, R. C. \& Carnevale, G. F. 2008 Vertical scale selection in inertial instability. J. Fluid Mech. 594 (1), 249-269.

Kloosterziel, R. C., Carnevale, G. F. \& Orlandi, P. 2007 Inertial instability in rotating and stratified fluids: barotropic vortices. J. Fluid Mech. 583, 379-412.

Kloosterziel, R. C. \& VAN Heisst, G. J. F. 1991 An experimental study of unstable barotropic vortices in a rotating fluid. J. Fluid Mech. 223, 1-24.

Lazar, A., Stegner, A., Caldeira, R., Dong, C., Didelle, H. \& Viboud, S. 2013 Inertial instability of intense stratified anticyclones. Part 2. Laboratory experiments. J. Fluid Mech. 732, 485-509.

Leblanc, S. \& CAMBON, C. 1997 On the three-dimensional instabilities of plane flows subjected to Coriolis force. Phys. Fluids 9 (5), 1307-1316. 
Mutabazi, I., Normand, C. \& Wesfreid, J. E. 1992 Gap size effects on centrifugally and rotationally driven instabilities. Phys. Fluids A 4, 1199-1205.

OOYAMA, K. 1966 On the stability of the baroclinic circular vortex: a sufficient criterion for instability. J. Atmos. Sci. 23, 43-53.

Orlandi, P. \& Carnevale, G. F. 1999 Evolution of isolated vortices in a rotating fluid of finite depth. J. Fluid Mech. 381, 239-269.

Plougonven, R. \& Zeitlin, V. 2009 Nonlinear development of inertial instability in a barotropic shear. Phys. Fluids 21 (10), 106601.

SAWYer, J. S. 1947 Notes on the theory of tropical cyclones. Q. J. R. Meteorol. Soc. 73 (315/316), 101-126.

SIPP, D. \& JACQUIN, L. 2000 Three-dimensional centrifugal-type instabilities of two dimensional flows in rotating systems. Phys. Fluids 12 (7), 1740-1748.

Smyth, W. D. \& MCWilliams, J. C. 1998 Instability of an axisymmetric vortex in a stably stratified, rotating environment. Theor. Comput. Fluid Dyn. 11 (3/4), 305-322.

Stevens, D. E. \& Ciesielski, P. E. 1986 Inertial instability of horizontally sheared flow away from the equator. J. Atmos. Sci. 43 (23), 2845-2856.

Stone, P. H. 1966 On non-geostrophic baroclinic stability. J. Atmos. Sci. 23, 390-400.

TAYLOR, J. R. \& FERRARI, R. 2009 On the equilibration of a symmetrically unstable front via a secondary shear instability. J. Fluid Mech. 622, 103-113.

Teinturier, S., Stegner, A., Didelle, H. \& Viboud, S. 2010 Small-scale instabilities of an island wake flow in a rotating shallow-water layer. Dyn. Atmos. Oceans 49, 1-24.

Yanase, S., Flores, C., Métais, O. \& Riley, J. J. 1993 Rotating free-shear flows. Part 1. Linear stability analysis. Phys. Fluids A 5, 2725-2737. 